Ann. Inst. Henri Poincaré Comb. Phys. Interact. 5 (2018), 437-466

DOI 10.4171/AIHPD/59

\title{
Moments of quantum Lévy areas using sticky shuffle Hopf algebras
}

\author{
Robin Hudson, Uwe Schauz, and Yue Wu
}

\begin{abstract}
We study a family of quantum analogs of Lévy's stochastic area for planar Brownian motion depending on a variance parameter $\sigma \geq 1$ which deform to the classical Lévy area as $\sigma \rightarrow \infty$. They are defined as second rank iterated stochastic integrals against the components of planar Brownian motion, which are one-dimensional Brownian motions satisfying Heisenberg-type commutation relations. Such iterated integrals can be multiplied using the sticky shuffle product determined by the underlying Itô algebra of stochastic differentials. We use the corresponding Hopf algebra structure to evaluate the moments of the quantum Lévy areas and study how they deform to their classical values, which are well known to be given essentially by the Euler numbers, in the infinite variance limit.
\end{abstract}

Mathematics Subject Classification (2010). 81S25, 46L53.

Keywords. Lévy area, non-Fock quantum stochastic calculus, moments, sticky shuffles, Euler numbers.

\section{Contents}

1 Introduction . . . . . . . . . . . . . . . . . 438

2 The sticky shuffle product Hopf algebra . . . . . . . . . . . . . . . 441

3 Moments and sticky shuffles . . . . . . . . . . . . . . . . . 445

4 Some background about Eulerian and Euler numbers . . . . . . . . . . 451

5 Moments of quantum Lévy areas . . . . . . . . . . . . . . . . . . . . . . . . . . . . . . . . . . . . .

6 The classical limit . . . . . . . . . . . . . . . . . . . 464

References ....................... 465 


\section{Introduction}

Lévy's stochastic area for planar Brownian motion is important in several areas of modern mathematics and probability theory, ranging from harmonic analysis on the Heisenberg group to rough noise analysis.

Let us first review the definition of Lévy area as a stochastic integral [13]. Intuitively it is the signed area between the chord joining two time points on the planar Brownian path and the trajectory between those points. To make this rigorous, let there be given a planar Brownian motion $B$ and write $B=(X, Y)$ in terms of components $X$ and $Y$ which are independent one-dimensional Brownian motions. Let two real numbers $a<b$ be given.

Definition 1. The Lévy area of $B$ over the time interval $[a, b)$ is the stochastic integral

$$
\mathcal{A}_{[a, b)}=\frac{1}{2} \int_{a}^{b}((X-X(a)) d Y-(Y-Y(a)) d X) .
$$

In this definition the integral takes the same value whether it is regarded as of Stratonovich or Itô type. In the remainder of this paper, however, all stochastic integrals will be of Itô type, in contrast to [11] where the Stratonovich integral is used. The latter cannot be defined coherently in a quantum context.

Lévy's area has interesting connections with classical mathematics through its characteristic function, which is given by the following theorem.

Theorem 2 (Lévy [12]). We have

$$
\mathbb{E}\left[\exp \left(i z \mathcal{A}_{[a, b)}\right)\right]=\operatorname{sech}\left(\frac{1}{2}(b-a) z\right) .
$$

We can expand the right-hand side of the formula in Theorem 2 using the Taylor series

$$
\operatorname{sech}(z)=\sum_{m=0}^{\infty}(-1)^{m} \frac{A_{2 m}}{(2 m) !} z^{2 m},
$$

where the even Euler zigzag numbers $A_{2 m}$ are related to the Riemann zeta function $\zeta$ by

$$
\zeta(2 m)=\frac{\pi^{2 m}}{(2 m) !} A_{2 m} .
$$

Levin and Wildon in [11] used iterated integrals and combinatorial arguments arising from the formalism of rough noise to evaluate the moments of $\mathcal{A}_{[0,1)}$, which is tantamount to proving Theorem 2 . 
A one-parameter family of quantum Lévy areas has been introduced recently $[7,3]$. In these the component one-dimensional Brownian motions of the classical Lévy area are replaced by a pair of self adjoint operator-valued processes $\left(P^{(\sigma)}(t), Q^{(\sigma)}(t)\right)_{t \geq 0}$. Each such pair is determined by a variance parameter $\sigma$ taking a value in the range $1 \leq \sigma<\infty$. Each of $P^{(\sigma)}$ and $Q^{(\sigma)}$ is individually a one-dimensional Brownian motion of variance $\sigma^{2}$, so that for example, for each positive time $t, P^{(\sigma)}(t)$ is a Gaussian random variable of mean 0 and variance $\sigma^{2} t$. But the processes $P^{(\sigma)}$ and $Q^{(\sigma)}$ do not commute with each other; instead they satisfy the Heisenberg type commutation relation

$$
\left[P^{(\sigma)}(s), Q^{(\sigma)}(t)\right]=-2 i \min \{s, t\}
$$

in the rigorous Weyl sense that for arbitrary real $x$ and $y$ and nonnegative $s$ and $t$,

$$
e^{i x P^{(\sigma)}(s)} e^{i y Q^{(\sigma)}(t)}=e^{2 i x y \min \{s, t\}} e^{i y Q^{(\sigma)}(t)} e^{i x P^{(\sigma)}(s)}
$$

as unitary operators. Despite their mutual noncommutativity $P^{(\sigma)}$ and $Q^{(\sigma)}$ can be regarded as stochastically independent in a certain sense, and hence as the two components of a quantum planar Brownian motion. Indeed, for arbitrary real $x_{1}, x_{2}, \ldots, x_{m}, y_{1}, y_{2}, \ldots, y_{n}$ and nonnegative $s_{1}, s_{2}, \ldots, s_{m}, t_{1}, t_{2}, \ldots, t_{n}$, the operator $\sum x_{j} P^{(\sigma)}\left(s_{j}\right)+\sum y_{k} Q^{(\sigma)}\left(t_{k}\right)$ defined on the intersection of domains is essentially self-adjoint, so that the quantum probabilistic expectation $\mathbb{E}\left[e^{i\left(\sum x_{j} P^{(\sigma)}\left(s_{j}\right)+\sum y_{k} Q^{(\sigma)}\left(t_{k}\right)\right)}\right]$, which in effect determines the joint characteristic function, is well defined. Moreover this factorizes:

$$
\begin{aligned}
& \mathbb{E}\left[e^{i\left(\sum x_{j} P^{(\sigma)}\left(s_{j}\right)+\sum y_{k} Q^{(\sigma)}\left(t_{k}\right)\right)}\right] \\
& \quad=\mathbb{E}\left[e^{i \sum x_{j} P^{(\sigma)}\left(s_{j}\right)}\right] \mathbb{E}\left[e^{i \sum y_{k} Q^{(\sigma)}\left(t_{k}\right)}\right]
\end{aligned}
$$

and in classical probability such factorization is sufficient for independence.

We use the standard quantum stochastic calculus of [15] in the case when $\sigma=1$ and the non-Fock finite temperature calculus of [8] when $\sigma>1$ to define the corresponding quantum Lévy areas, in which the planar Brownian motion is replaced by its quantum version $R^{(\sigma)}=\left(P^{(\sigma)}, Q^{(\sigma)}\right)$.

Definition 3. The quantum Lévy area $\mathcal{B}_{[a, b)}^{(\sigma)}$ of $R^{(\sigma)}$ of variance $\sigma$ over the time interval $[a, b)$ is the quantum stochastic integral

$$
\mathcal{B}_{[a, b)}^{(\sigma)}=\frac{1}{2} \int_{a}^{b}\left(\left(P^{(\sigma)}-P^{(\sigma)}(a)\right) d Q^{(\sigma)}-\left(Q^{(\sigma)}-Q^{(\sigma)}(a)\right) d P^{(\sigma)}\right) .
$$


When $\sigma=1$, the distribution at all times of the corresponding Lévy areas is degenerate at 0 and all moments are zero [3]. For values $\sigma>1$ the processes $R^{(\sigma)}$ generate Type III factorial von Neumann algebras, ${ }^{1}$ whose mutual strong unitary inequivalence as $\sigma$ varies can be regarded as a quantum version of the mutual singularity of the measures obtained by dilatation of planar Wiener measure through different dilatation factors $\sigma$.

In view of (3), the normalised standard unit variance Brownian motions

$$
\hat{P}^{(\sigma)}=\sigma^{-1} P^{(\sigma)}, \quad \hat{Q}^{(\sigma)}=\sigma^{-1} Q^{(\sigma)},
$$

become mutually commutative in the limit of large $\sigma$, so that the corresponding quantum Lévy areas

$$
\widehat{\mathcal{B}}_{[a, b)}^{(\sigma)}=\sigma^{-2} \mathcal{B}_{[a, b)}^{(\sigma)}
$$

interpolate between the degenerate distribution at $\sigma=1$ and the classical case $\mathcal{A}_{[a, b)}$ at $\infty$. Thus it is a natural question to ask how the moments behave under this interpolation and in particular how the Euler zigzag numbers are approached at $\infty$. The main purpose of this paper is to address this question.

Our method is based firstly on the observation that Definition 1, Definition 3, and the normalized form of the latter, can be regarded as iterated stochastic integrals:

$$
\begin{aligned}
& \mathcal{A}_{[a, b)}=\frac{1}{2} \int_{a<x<y<b}(d X(x) d Y(y)-d Y(x) d X(y)), \\
& \mathcal{B}_{[a, b)}^{(\sigma)}=\frac{1}{2} \int_{a<x<y<b}\left(d P^{(\sigma)}(x) d Q^{(\sigma)}(y)-d Q^{(\sigma)}(x) d P^{(\sigma)}(y)\right), \\
& \hat{\mathcal{B}}_{[a, b)}^{(\sigma)}=\frac{1}{2} \int_{a<x<y<b}\left(d \hat{P}^{(\sigma)}(x) d \hat{Q}^{(\sigma)}(y)-d \hat{Q}^{(\sigma)}(x) d \hat{P}^{(\sigma)}(y)\right) .
\end{aligned}
$$

We may thus evaluate moments as expectations of powers, using the so-called sticky shuffle [6] or stuffle [5] Hopf algebra. Multiplication in this algebra can be used to express the product of two iterated Itô stochastic integrals as a linear combination of such iterated integrals. Since the expectation of an iterated integral vanishes unless each of the individual integrators is time, the recovery formula $[6,1]$, involving higher order Hopf algebra coproducts, reduces the evaluation of the moments to a combinatorial counting problem.

\footnotetext{
${ }^{1}$ More precisely, it is the the unitary operators $e^{i x P^{(\sigma)}(s)}$ and $e^{i x Q^{(\sigma)}(s)}$ for real $x$ and positive $\sigma$ which generate these algebras.
} 
As mentioned above, the moments of Levy area are directly related to classical formulas of Euler for values of the zeta function at even integers. Many more modern applications depend on the so-called Levy area formula [12] for the conditional characteristic function given the the values of the increments. Among many recent results of this type are proofs of Apery's theorem and more general results on values of the zeta function at odd integers, and also new results on values of multizeta functions. Because of mutual noncommutativity analogous joint conditioning cannot be applied to the component processes of the quantum Levy processes considered here. Instead, motivated by Yor's conceptual simplification of the conditional characteristic function using the rotational symmetry of planar Brownian motion, which is shared in a certain sense by the quantum planar Brownian motions considered here, it may be interesting to study what amounts to a joint characteristic function for quantum Levy areas with its "radial part" $P(t)^{2}+Q(t)^{2}$ by regarding the latter as an iterated quantum stochastic integral. Other quantum "quadratic Wiener functionals," with their many mathematical links, may also be explored. A start in this exploration has been made in [10].

The sticky shuffle Hopf algebra is reviewed in Section 2. Its use for reducing the evaluation of moments to a counting problem is described in Section 3. Several combinatorial results needed to accomplish the nontrivial counting task are then provided in Section 4. These combinatorial results are crucial within our calculations, but may become useful beyond the scope of this paper, too. In Section 5, we evaluate the moments of the quantum Lévy area (10). Finally, in Section 6, we show how the classical moments [11] are recovered in the "infinite temperature" limit as $\sigma \rightarrow \infty$.

\section{The sticky shuffle product Hopf algebra}

2.1. The shuffle product Hopf algebra. Given a complex vector space $\mathcal{L}$, the usual shuffle product Hopf algebra over $\mathcal{L}$ is formed by equipping the vector space $\mathcal{T}(\mathcal{L})=\bigoplus_{n=0}^{\infty} \bigotimes_{j=1}^{n} \mathcal{L}$ of tensors of all ranks over $\mathcal{L}$ with the operations of product, unit, coproduct, counit and antipode defined as follows. We denote a general element $\alpha$ of $\mathcal{T}(\mathcal{L})$ by $\alpha=\alpha_{0} \oplus \alpha_{1} \oplus \alpha_{2} \oplus \cdots$ or $\left(\alpha_{0}, \alpha_{1}, \alpha_{2}, \ldots\right)$, where only finitely many of the $\alpha_{m}$ are nonzero. For each $\alpha_{m} \in \bigotimes_{j=1}^{m} \mathcal{L}$ the corresponding embedded element $\left(0,0, \ldots, \alpha_{m}, 0, \ldots\right)$ of $\mathcal{T}(\mathcal{L})$ is denoted by $\left\{\alpha_{m}\right\}$. 
- The shuffle product is defined by bilinear extension of the rule

$$
\begin{aligned}
& \left\{L_{1} \otimes L_{2} \otimes \cdots \otimes L_{m}\right\}\left\{L_{m+1} \otimes L_{m+2} \otimes \cdots \otimes L_{m+n}\right\} \\
& \quad=\sum_{\mathfrak{s} \in \mathcal{S}(m, n)}\left\{L_{\mathfrak{s}(1)} \otimes L_{\mathfrak{s}(2)} \otimes \cdots \otimes L_{\mathfrak{s}(m+n)}\right\}
\end{aligned}
$$

where $\mathcal{S}(m, n)$ denotes the set of $(m, n)$-shuffles, that is permutations $\mathfrak{s}$ of $\{1,2, \ldots, m+n\}$ for which $\mathfrak{s}(1)<\mathfrak{s}(2)<\cdots<\mathfrak{s}(m)$ and $\mathfrak{s}(m+1)<$ $\mathfrak{s}(m+2)<\cdots<\mathfrak{s}(m+n)$.

- The unit element for this product is $1_{\mathcal{T}(\mathcal{L})}=\left(1_{\mathbb{C}}, 0,0, \ldots\right)$.

- The coproduct $\Delta$ is the map from $\mathcal{T}(\mathcal{L})$ to $\mathcal{T}(\mathcal{L}) \otimes \mathcal{T}(\mathcal{L})$ defined by linear extension of the rules that $\Delta\left(1_{\mathcal{T}(\mathcal{L})}\right)=1_{\mathcal{T}(\mathcal{L})} \otimes 1_{\mathcal{T}(\mathcal{L})}=1_{\mathcal{T}(\mathcal{L}) \otimes \mathcal{T}(\mathcal{L})}$ and

$$
\begin{aligned}
& \Delta\left\{L_{1} \otimes L_{2} \otimes \cdots \otimes L_{m}\right\} \\
& =1_{\mathcal{T}(\mathcal{L})} \otimes\left\{L_{1} \otimes L_{2} \otimes \cdots \otimes L_{m}\right\} \\
& \quad+\sum_{j=2}^{m}\left\{L_{1} \otimes L_{2} \otimes \cdots \otimes L_{j-1}\right\} \otimes\left\{L_{j} \otimes L_{j+1} \otimes \cdots \otimes L_{m}\right\} \\
& \quad+\left\{L_{1} \otimes L_{2} \otimes \cdots \otimes L_{m}\right\} \otimes 1_{\mathcal{T}(\mathcal{L}) .}
\end{aligned}
$$

- The counit $\varepsilon$ is the map from $\mathcal{T}(\mathcal{L})$ to $\mathbb{C}$ defined by linear extension of

$$
\varepsilon\left(1_{\mathcal{T}(\mathcal{L})}\right)=1_{\mathbb{C}}, \varepsilon\left\{L_{1} \otimes L_{2} \otimes \cdots \otimes L_{m}\right\}=0 \quad \text { for } m>0 .
$$

- The antipode is the map $S$ from $\mathcal{T}(\mathcal{L})$ to $\mathcal{T}(\mathcal{L})$ defined by linear extension of

$$
S\left(1_{\mathcal{T}(\mathcal{L})}\right)=1_{\mathcal{T}(\mathcal{L})}
$$

and

$$
S\left\{L_{1} \otimes L_{2} \otimes \cdots \otimes L_{m}\right\}=(-1)^{m}\left\{L_{m} \otimes L_{m-1} \otimes \cdots \otimes L_{1}\right\}
$$

for $m>0$.

There are two useful equivalent definitions of the shuffle product. For the first, we use the notational convention that, for arbitrary elements $\alpha$ of $\mathcal{T}(\mathcal{L})$ and $L$ of $\mathcal{L}, \alpha \otimes L$ is the element of $\mathcal{T}(\mathcal{L})$ for which $(\alpha \otimes L)_{0}=0$ and $(\alpha \otimes L)_{n}=\alpha_{n-1} \otimes L$ for $n \geq 1$. Then the shuffle product of arbitrary elements of $\mathcal{T}(\mathcal{L})$ is defined inductively by bilinear extension of the rules

$$
\begin{aligned}
1_{\mathcal{T}(\mathcal{L})}\left\{L_{1} \otimes L_{2} \otimes \cdots \otimes L_{m}\right\} & =\left\{L_{1} \otimes L_{2} \otimes \cdots \otimes L_{m}\right\} 1_{\mathcal{T}(\mathcal{L})} \\
& =\left\{L_{1} \otimes L_{2} \otimes \cdots \otimes L_{m}\right\},
\end{aligned}
$$


and

$$
\begin{aligned}
& \left\{L_{1} \otimes L_{2} \otimes \cdots \otimes L_{m}\right\}\left\{L_{m+1} \otimes L_{m+2} \otimes \cdots \otimes L_{m+n}\right\} \\
& =\left(\left\{L_{1} \otimes L_{2} \otimes \cdots \otimes L_{m-1}\right\}\left\{L_{m+1} \otimes L_{m+2} \otimes \cdots \otimes L_{m+n}\right\}\right) \otimes L_{m} \\
& \quad+\left(\left\{L_{1} \otimes L_{2} \otimes \cdots \otimes L_{m}\right\}\left\{L_{m+1} \otimes L_{m+2} \otimes \cdots \otimes L_{m+n-1}\right\}\right) \otimes L_{m+n} .
\end{aligned}
$$

Here the two terms on the right-hand side of (17) correspond to the mutually exclusive and exhaustive possibilities that $\mathfrak{s}(m+n)=m$ and $\mathfrak{s}(m+n)=m+n$ in the expansion (11). The second alternative definition is that the shuffle product $\gamma=\alpha \beta$ of arbitrary elements $\alpha$ and $\beta$ is given by

$$
\begin{gathered}
\gamma_{N}=\sum_{\substack{A \cup B=\{1,2, \ldots, N\} \\
A \cap B=\emptyset}} \alpha_{|A|}^{A} \beta_{|B|}^{B} . \\
.
\end{gathered}
$$

Here the sum is over the $2^{N}$ ordered pairs $(A, B)$ of disjoint subsets whose union is $\{1,2, \ldots, N\}$ and the notation is as follows; $|A|$ denotes the number of elements in the set $A$ so that $\alpha_{|A|}$ denotes the homogeneous component of rank $|A|$ of the tensor $\alpha=\left(\alpha_{0}, \alpha_{1}, \alpha_{2}, \ldots\right)$, and $\alpha_{|A|}^{A}$ indicates that this component is to be regarded as occupying only those $|A|$ copies of $\mathcal{L}$ within $\otimes_{j=1}^{N} \mathcal{L}$ labelled by elements of the subset $A$ of $\{1,2, \ldots, N\}$. Thus with $\beta_{|B|}^{B}$ defined analogously the combination $\alpha_{|A|}^{A} \beta_{|B|}^{B}$ is a well-defined element of $\bigotimes_{j=1}^{N} \mathcal{L}$.

2.2. The sticky shuffle algebra. Now suppose that the complex vector space $\mathcal{L}$ is an associative algebra. We define the sticky shuffle product in the vector space $\mathcal{T}(\mathcal{L})$ by modifying definition $(17)$ by inserting an extra term so that now

$$
\begin{aligned}
& \left\{L_{1} \otimes L_{2} \otimes \cdots \otimes L_{m}\right\}\left\{L_{m+1} \otimes L_{m+2} \otimes \cdots \otimes L_{m+n}\right\} \\
& =\left(\left\{L_{1} \otimes \cdots \otimes L_{m-1}\right\}\left\{L_{m+1} \otimes \cdots \otimes L_{m+n}\right\}\right) \otimes L_{m} \\
& \quad+\left(\left\{L_{1} \otimes \cdots \otimes L_{m}\right\}\left\{L_{m+1} \otimes \cdots \otimes L_{m+n-1}\right\}\right) \otimes L_{m+n} \\
& \quad+\left(\left\{L_{1} \otimes \cdots \otimes L_{m-1}\right\}\left\{L_{m+1} \otimes \cdots \otimes L_{m+n-1}\right\}\right) \otimes L_{m} L_{m+n} .
\end{aligned}
$$

The sticky shuffle (also known as quasishuffle and as stuffle) Hopf algebra appears to originate in [14]. Or we can modify the alternative definition of the shuffle product (18) by defining the product $\gamma=\alpha \beta$ by

$$
\gamma_{N}=\sum_{A \cup B=\{1,2, \ldots, N\}} \alpha_{|A|}^{A} \beta_{|B|}^{B} .
$$


Here the sum is now over the $3^{N}$ not necessarily disjoint ordered pairs $(A, B)$ whose union is $\{1,2, \ldots, N\}, \alpha_{|A|}^{A}$ and $\beta_{|B|}^{B}$ are defined as before but now if $A \cap B \neq \emptyset$ double occupancy of a copy of $\mathcal{L}$ within $\bigotimes_{j=1}^{n} \mathcal{L}$ is reduced to single occupancy by using the multiplication in the algebra $\mathcal{L}$ as a map from $\mathcal{L} \times \mathcal{L}$ to $\mathcal{L}$. Thus the sticky shuffle product reduces to the usual shuffle product in the case when the multiplication in $\mathcal{L}$ is trivial with all products vanishing. That (20) is equivalent to (19) (and in particular, that (18) is equivalent to (17)) is seen by noting that the three terms on the right-hand side of (19) correspond to the three mutually exclusive and exhaustive possibilities that $N \in A \cap B^{c}, N \in A^{c} \cap B$ and $N \in A \cap B$ in (20).

The same unit, coproduct and counit as before can be applied to make the sticky shuffle product algebra into a Hopf algebra, but the definition of the antipode must be modified [6] to

$$
\begin{gathered}
(-1)^{m} S\left\{L_{1} \otimes L_{2} \otimes \cdots \otimes L_{m}\right\} \\
=\left\{L_{m} \otimes L_{m-1} \otimes \cdots \otimes L_{1}\right\} \\
+\sum_{r=1}^{m} \sum_{1 \leq k_{1}<k_{2}<\cdots<k_{r-1}<m}\left\{L_{k_{r-1}+1} L_{k_{r-1}+2} \ldots L_{m}\right. \\
\stackrel{L_{k_{r-2}+1} L_{k_{r-2}+2} \ldots L_{k_{r-1}} \otimes \cdots}{\left.\otimes L_{1} L_{2} \ldots L_{k_{1}}\right\} .}
\end{gathered}
$$

The recovery formula [1] expresses the homogeneous components of an element $\alpha$ of $\mathcal{T}(\mathcal{L})$ in terms of the iterated coproduct $\Delta^{(N)} \alpha$ by

$$
\alpha_{N}=\left(\Delta^{(N)} \alpha\right)_{(1,1, \ldots, 1}^{(N)},{ }^{(N)}
$$

Here, $\Delta^{(N)}$ is defined recursively by

$$
\Delta^{(2)}=\Delta \quad \text { and } \quad \Delta^{(N)}=\left(\Delta \otimes \operatorname{Id}_{\otimes_{(N-2)} \mathcal{T}(\mathcal{L})}\right) \circ \Delta^{(N-1)} \quad \text { for } N>2 .
$$

Hence, it is a map from $\mathcal{T}(\mathcal{L})$ to the $N$ th tensor power

$$
\bigotimes^{(N)} \mathcal{T}(\mathcal{L})=\bigotimes^{(N)} \bigoplus_{n=0}^{\infty} \bigotimes_{j=1}^{n} \mathcal{L}=\bigoplus_{n_{1}, n_{2}, \ldots, n_{N}=0}^{\infty} \bigotimes_{r=1}^{N} \bigotimes_{j_{r}=1}^{n_{r}} \mathcal{L}
$$

so that $\Delta^{(N)} \alpha$ has multirank components $\alpha_{\left(n_{1}, n_{2}, \ldots, n_{N}\right)}$ of all orders. The recovery formula (21) also holds when $N=0$ and $N=1$ if we define $\Delta^{(0)}$ and $\Delta^{(1)}$ to be the counit $\varepsilon$ and the identity map $\operatorname{Id}_{\mathcal{T}(\mathcal{L})}$ respectively. 
Note that the recovery formula is the same for both the sticky and nonsticky cases; it only involves the coproduct $\Delta$ which is one and the same map. However our application of it will use the fact that $\Delta$ is multiplicative, $\Delta(\alpha \beta)=\Delta(\alpha) \Delta(\beta)$, where the product on the tensor square $\mathcal{T}(\mathcal{L}) \otimes \mathcal{T}(\mathcal{L})$ is defined by linear extension of the rule

$$
\left(a \otimes a^{\prime}\right)\left(b \otimes b^{\prime}\right)=a b \otimes a^{\prime} b^{\prime} .
$$

This holds in particular with the sticky shuffle product as the product in $\mathcal{T}(\mathcal{L})$.

\section{Moments and sticky shuffles}

We now describe the connection between sticky shuffle products and iterated stochastic integrals. We begin with the well-known fact that, for the one-dimensional Brownian motion $X$ and for $a \leq b$,

$$
(X(b)-X(a))^{2}=2 \int_{a \leq x<b}(X(x)-X(a)) d X(x)+\int_{a \leq x<b} d T(x),
$$

where $T(x)=x$ is time. We introduce the Itô algebra $\mathcal{L}=\mathbb{C}\langle d X, d T\rangle$ of complex linear combinations of the basic differentials $d X$ and $d T$, which are multiplied according to the table

\begin{tabular}{c|cc} 
& $d X$ & $d T$ \\
\hline$d X$ & $d T$ & 0 \\
$d T$ & 0 & 0
\end{tabular}

together with the corresponding sticky shuffle Hopf algebra $\mathcal{T}(\mathcal{L})$. For each pair of real numbers $a<b$, we introduce a map $J_{a}^{b}$ from $\mathcal{T}(\mathcal{L})$ to complex-valued random variables on the probability space of the Brownian motion $X$ by linear extension of the rule that, for arbitrary $d L_{1}, d L_{2}, \cdots d L_{m} \in\{d X, d T\}$

$$
\begin{aligned}
J_{a}^{b} & \left\{d L_{1} \otimes d L_{2} \otimes \cdots \otimes d L_{m}\right\} \\
& =\int_{a \leq x_{1}<x_{2}<\cdots<x_{m}<b} d L_{1}\left(x_{1}\right) d L_{2}\left(x_{2}\right) d L_{3}\left(x_{3}\right) \cdots d L_{m}\left(x_{m}\right) \\
& =\int_{a}^{b} \cdots \int_{a}^{x_{4}} \int_{a}^{x_{3}} \int_{a}^{x_{2}} d L_{1}\left(x_{1}\right) d L_{2}\left(x_{2}\right) d L_{3}\left(x_{3}\right) \cdots d L_{m}\left(x_{m}\right) .
\end{aligned}
$$

By convention $J_{a}^{b}$ maps the unit element of the algebra $\mathcal{T}(\mathcal{L})$ to the unit random variable identically equal to 1 . 
Then (26) can be restated as follows,

$$
J_{a}^{b}(\{d X\}) J_{a}^{b}(\{d X\})=J_{a}^{b}(\{d X\}\{d X\}),
$$

using the fact that $\{d X\}^{2}=2\{d X \otimes d X\}+\{d T\}$.

The following more general Theorem is probably known to many classical and quantum probabilists; the quantum version was first given in [2].

Theorem 4. For arbitrary $\alpha$ and $\beta$ in $\mathcal{T}(\mathcal{L})$,

$$
J_{a}^{b}(\alpha) J_{a}^{b}(\beta)=J_{a}^{b}(\alpha \beta) .
$$

Proof. By bilinearity it is sufficient to consider the case when

$$
\alpha=\left\{d L_{1} \otimes d L_{2} \otimes \cdots \otimes d L_{m}\right\}, \quad \beta=\left\{d L_{m+1} \otimes d L_{m+2} \otimes \cdots \otimes d L_{m+n}\right\}
$$

for $d L_{1}, d L_{2}, \cdots, d L_{m+n} \in\{d X, d T\}$. In this case Theorem 4 follows, using the inductive definition (19) for the sticky shuffle product, from the product form of Itô's formula,

$$
d(\xi \eta)=(d \xi) \eta+\xi d \eta+(d \xi) d \eta
$$

where stochastic differentials of the form $d \xi=F d X+G d T$, with stochastically integrable processes $F$ and $G$, are multiplied using table (27).

For planar Brownian motion $R=(X, Y)$ the Ito table (27) becomes

\begin{tabular}{c|ccc} 
& $d X$ & $d Y$ & $d T$ \\
\hline$d X$ & $d T$ & 0 & 0 \\
$d Y$ & 0 & $d T$ & 0 \\
$d T$ & 0 & 0 & 0
\end{tabular}

For the quantum planar Brownian motion $\left(P^{(\sigma)}, Q^{(\sigma)}\right)$ it becomes

\begin{tabular}{c|ccc} 
& $d P^{(\sigma)}$ & $d Q^{(\sigma)}$ & $d T$ \\
\hline$d P^{(\sigma)}$ & $\sigma^{2} d T$ & $-i d T$ & 0 \\
$d Q^{(\sigma)}$ & $i d T$ & $\sigma^{2} d T$ & 0 \\
$d T$ & 0 & 0 & 0
\end{tabular}

Theorem 5. Theorem 4 holds when $\mathcal{L}$ is either of the algebras defined by the multiplication tables (32) and (33).

Remark 6. In both cases this follows from the corresponding Itô product rule (31). For classical planar Brownian motion the Itô product rule is well-known. For the quantum case, when $\sigma=1$ see [15] and when $\sigma>1$, see [8]. 
In view of (8) and (9)

$$
\begin{aligned}
& \mathcal{A}_{[a, b)}=\frac{1}{2} J_{a}^{b}(d X \otimes d Y-d Y \otimes d X), \\
& \mathcal{B}_{[a, b)}^{(\sigma)}=\frac{1}{2} J_{a}^{b}\left(d P^{(\sigma)} \otimes d Q^{(\sigma)}-d Q^{(\sigma)} \otimes d P^{(\sigma)}\right)
\end{aligned}
$$

For use below we note that the table (32) becomes

\begin{tabular}{c|ccc} 
& $d Z$ & $d \bar{Z}$ & $d T$ \\
\hline$d Z$ & 0 & $\frac{1}{2} d T$ & 0 \\
$d \bar{Z}$ & $\frac{1}{2} d T$ & 0 & 0 \\
$d T$ & 0 & 0 & 0
\end{tabular}

in terms of the basis $(d Z, d \bar{Z}, d T)$ where

$$
d Z=\frac{1}{2}(-i d X+d Y), \quad d \bar{Z}=\frac{1}{2}(i d X+d Y) .
$$

Correspondingly

$$
\mathcal{A}_{[a, b)}=i J_{a}^{b}(d Z \otimes d \bar{Z}-d \bar{Z} \otimes d Z)
$$

Similarly (33) becomes

\begin{tabular}{c|ccc} 
& $d A^{(\sigma)}$ & $d A^{\dagger(\sigma)}$ & $d T$ \\
\hline$d A^{(\sigma)}$ & 0 & $\frac{1}{2}\left(\sigma^{2}+1\right) d T$ & 0 \\
$d A^{\dagger(\sigma)}$ & $\frac{1}{2}\left(\sigma^{2}-1\right) d T$ & 0 & 0 \\
$d T$ & 0 & 0 & 0
\end{tabular}

in terms of the basis $\left(d A^{(\sigma)}, d A^{\dagger(\sigma)}, d T\right)$ where

$$
d A^{(\sigma)}=\frac{1}{2}\left(-i d P^{(\sigma)}+d Q^{(\sigma)}\right), d A^{\dagger(\sigma)}=\frac{1}{2}\left(i d P^{(\sigma)}+d Q^{(\sigma)}\right) .
$$

For the basis $\left(d \hat{P}^{(\sigma)}, d \hat{Q}^{(\sigma)}, d T\right),(33)$ becomes

\begin{tabular}{c|ccc} 
& $d \hat{P}^{(\sigma)}$ & $d \hat{Q}^{(\sigma)}$ & $d T$ \\
\hline$d \hat{P}^{(\sigma)}$ & $d T$ & $-i \sigma^{-2} d T$ & 0 \\
$d \hat{Q}^{(\sigma)}$ & $i \sigma^{-2} d T$ & $d T$ & 0 \\
$d T$ & 0 & 0 & 0
\end{tabular}

which deforms to the classical table (32) as $\sigma \rightarrow \infty$. Similarly, for the basis $\left(d \widehat{A}^{(\sigma)}, d \hat{A}^{\dagger(\sigma)}, d T\right)$ where

$$
d \widehat{A}^{(\sigma)}=\frac{1}{2}\left(-i d \hat{P}^{(\sigma)}+d \hat{Q}^{(\sigma)}\right), d \hat{A}^{\dagger(\sigma)}=\frac{1}{2}\left(i d \hat{P}^{(\sigma)}+d \widehat{Q}^{(\sigma)}\right)
$$


we have

\begin{tabular}{c|ccc} 
& $d \widehat{A}^{(\sigma)}$ & $d \hat{A}^{\dagger(\sigma)}$ & $d T$ \\
\hline$d \hat{A}^{(\sigma)}$ & 0 & $\sigma_{+} d T$ & 0 \\
$d \hat{A}^{\dagger(\sigma)}$ & $\sigma_{-} d T$ & 0 & 0 \\
$d T$ & 0 & 0 & 0
\end{tabular}

with

$$
\sigma_{ \pm}=\frac{1}{2}\left(1 \pm \sigma^{-2}\right)
$$

which becomes isomorphic to (36) when $\sigma \rightarrow \infty$. The normalized quantum Lévy area which is our main concern is

$$
\widehat{\mathcal{B}}_{[a, b)}^{(\sigma)}=i J_{a}^{b}\left(d \widehat{A}^{(\sigma)} \otimes d \hat{A}^{\dagger(\sigma)}-d \hat{A}^{\dagger(\sigma)} \otimes d \widehat{A}^{(\sigma)}\right) .
$$

In the following theorem, the basis referred to is any of those for which the respective algebras have multiplication tables (32), (33), (36), (39), (41) or (43).

Theorem 7. For arbitrary $n \in \mathbb{N}, a<b \in \mathbb{R}$ and basis elements $d L_{1}, d L_{2}, \ldots$, $d L_{n}$

$$
\mathbb{E}\left[J_{a}^{b}\left\{d L_{1} \otimes d L_{2} \otimes \cdots \otimes d L_{n}\right\}\right]=0
$$

unless

$$
d L_{1}=d L_{2}=\cdots=d L_{n}=d T \text {. }
$$

Proof. If $d L_{n} \neq d T$ then

$$
J_{a}^{b}\left\{d L_{1} \otimes d L_{2} \otimes \cdots \otimes d L_{n}\right\}=\int_{a}^{b} J_{a}^{x}\left\{d L_{1} \otimes d L_{2} \otimes \cdots \otimes d L_{n-1}\right\} d L_{n}(x) .
$$

In the classical cases (32) and (36), $L_{n}$ is a real or complex-valued martingale and the expectation of the stochastic integral against $d L_{n}$ vanishes. When $\sigma=1$ it also vanishes in the cases (33), (39) and (41) by the first fundamental formula of quantum stochastic calculus in the Fock space $\mathcal{F}$ [15]. When $\sigma>1$ it vanishes as may be seen for example by realising the processes $P^{(\sigma)}$ and $Q^{(\sigma)}$ in the tensor product of $\mathcal{F}$ with its Hilbert space dual, $\mathcal{F} \otimes \overline{\mathcal{F}}$, equipped with the tensor product $e(0) \otimes \overline{e(0)}$ of the Fock vacuum vector with its dual vector as

$$
\begin{aligned}
& P^{(\sigma)}=\sqrt{\frac{1}{2}\left(\sigma^{2}+1\right)}\left(P^{(\sigma)} \otimes \bar{I}\right)+\sqrt{\frac{1}{2}\left(\sigma^{2}-1\right)}\left(I \otimes \bar{P}^{(\sigma)}\right), \\
& Q^{(\sigma)}=\sqrt{\frac{1}{2}\left(\sigma^{2}+1\right)}\left(Q^{(\sigma)} \otimes \bar{I}\right)+\sqrt{\frac{1}{2}\left(\sigma^{2}-1\right)}\left(I \otimes \bar{Q}^{(\sigma)}\right),
\end{aligned}
$$


and again invoking the first fundamental formula. Thus in all cases

$$
\mathbb{E}\left[J_{a}^{b}\left\{d L_{1} \otimes d L_{2} \otimes \cdots \otimes d L_{n}\right\}\right]=0
$$

unless $d L_{n}=d T$.

If $d L_{n}=d T$ then by Fubini's theorem we can write

$$
\begin{aligned}
& \mathbb{E}\left[J_{a}^{b}\left\{d L_{1} \otimes d L_{2} \otimes \cdots \otimes d L_{n}\right\}\right] \\
& \quad=\int_{a}^{b}\left\{\mathbb{E}\left[J_{a}^{x}\left\{d L_{1} \otimes d L_{2} \otimes \cdots \otimes d L_{n-1}\right\}\right]\right\} d x \\
& =0
\end{aligned}
$$

unless $d L_{n-1}=d T$, by the previous argument. By repetition we see that

$$
\mathbb{E}\left[J_{a}^{b}\left\{d L_{1} \otimes d L_{2} \otimes \cdots \otimes d L_{n}\right\}\right]=0,
$$

unless each of $d L_{n}, d L_{n-1}, d L_{n-2}, \ldots, d L_{1}$ is equal to $d T$.

Now consider the moments sequence of the normalized quantum Lévy area of variance $\sigma^{2}$ in the form (45). In view of Theorem 4 ,

$$
\begin{aligned}
{\left[\hat{\mathcal{B}}_{[a, b)}^{(\sigma)}\right]^{n} } & =i^{n}\left(J_{a}^{b}\left(d \widehat{A}^{(\sigma)} \otimes d \hat{A}^{\dagger(\sigma)}-d \hat{A}^{\dagger(\sigma)} \otimes d \widehat{A}^{(\sigma)}\right)\right)^{n} \\
& =i^{n} J_{a}^{b}\left(\left\{d \widehat{A}^{(\sigma)} \otimes d \hat{A}^{\dagger(\sigma)}-d \hat{A}^{\dagger(\sigma)} \otimes d \widehat{A}^{(\sigma)}\right\}^{n}\right)
\end{aligned}
$$

The $n$th sticky shuffle power $\left\{d \widehat{A}^{(\sigma)} \otimes d \hat{A}^{\dagger(\sigma)}-d \hat{A}^{\dagger(\sigma)} \otimes d \widehat{A}^{(\sigma)}\right\}^{n}$ will consist of non-sticky shuffle products of rank $2 n$ together with terms of lower ranks $n, n+1, \ldots, 2 n-1$, all of which except the rank $n$ term will contain one or more copies of $d \hat{A}^{(\sigma)}$ and $d \hat{A}^{\dagger(\sigma)}$, and will thus not contribute to the expectation in view of Theorem 7. The term of rank $n$ will be a multiple of $d T \otimes d T \cdots \otimes \stackrel{(n)}{d T}$, where the symbol $(n)$ on the top of the last copy $d T$ indicates the position of the referred term in a row. Thus we can write

$$
\begin{aligned}
& \left\{d \widehat{A}^{(\sigma)} \otimes d \hat{A}^{\dagger(\sigma)}-d \hat{A}^{\dagger(\sigma)} \otimes d \widehat{A}^{(\sigma)}\right\}^{n} \\
& \quad=w_{n}^{(\sigma)}\{d T \otimes d T \cdots \otimes \stackrel{(n)}{d} T\}+\text { terms of rank }>n
\end{aligned}
$$


for some coefficient $w_{n}^{(\sigma)}$. The corresponding moment is given by

$$
\begin{aligned}
\mathbb{E}\left[\widehat{\mathcal{B}}_{[a, b]}^{(\sigma)}\right]^{n}= & i^{n} w_{n}^{(\sigma)} \mathbb{E}\left[J_{a}^{b}(\{d T \otimes d T \cdots \otimes d(n)\right. \\
= & i^{n} w_{n}^{(\sigma)} \int d x_{1} d x_{2} \ldots d x_{n} \\
& a \leq x_{1}<x_{2}<\cdots<x_{n}<b \\
= & i^{n} w_{n}^{(\sigma)} \frac{(b-a)^{n}}{n !}
\end{aligned}
$$

By the recovery formula (21) and the multiplicativity of the $n$th order coproduct $\Delta^{(n)}$,

$$
\begin{aligned}
& w_{n}^{(\sigma)} d T \otimes d T \cdots \otimes \stackrel{(n)}{d} T \\
& =\left\{\left\{d \widehat{A}^{(\sigma)} \otimes d \hat{A}^{\dagger(\sigma)}-d \hat{A}^{\dagger(\sigma)} \otimes d \widehat{A}^{(\sigma)}\right\}^{n}\right\}_{n} \\
& \left.=\left(\Delta^{(n)}\left(\left\{d \widehat{A}^{(\sigma)} \otimes d \hat{A}^{\dagger(\sigma)}-d \hat{A}^{\dagger(\sigma)} \otimes d \widehat{A}^{(\sigma)}\right\}^{n}\right)\right)_{(1,1, \ldots, 1} \underset{1}{(n)}\right)
\end{aligned}
$$

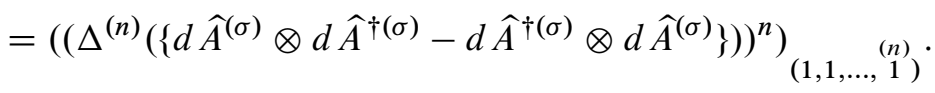

Now

$$
\begin{aligned}
& \Delta^{(n)}\left(\left\{d \widehat{A}^{(\sigma)} \otimes d \hat{A}^{\dagger(\sigma)}-d \hat{A}^{\dagger(\sigma)} \otimes d \widehat{A}^{(\sigma)}\right\}\right) \\
& =\sum_{1 \leq j \leq n} 1_{\mathcal{T}(\mathcal{L})} \otimes \cdots \otimes\left\{d \widehat{A}^{(\sigma)} \otimes d \widehat{A}^{\dagger(\sigma)}-d \hat{A}^{\dagger(\sigma)} \otimes d \widehat{A}^{(\sigma)}\right\} \otimes \cdots \otimes 1_{\mathcal{T}(\mathcal{L})} \\
& \quad+\sum_{1 \leq j<k \leq n}\left(1_{\mathcal{T}(\mathcal{L})} \otimes \cdots \otimes\left\{d \widehat{A}^{(\sigma)}\right\} \otimes \cdots \otimes\left\{d \hat{A}^{\dagger(\sigma)}\right\} \otimes \cdots \otimes 1_{\mathcal{T}(\mathcal{L})}^{(n)}\right. \\
& \left.\quad-1_{\mathcal{T}(\mathcal{L})} \otimes \cdots \otimes\left\{d \hat{A}^{\dagger(\sigma)}\right\} \otimes \cdots \otimes\left\{d \widehat{A}^{(\sigma)}\right\} \otimes \cdots \otimes 1_{\mathcal{T}(\mathcal{L})}^{(n)}\right) .
\end{aligned}
$$

The first term of this sum, being of rank 2, cannot contribute to the component of joint rank $(1,1, \ldots, \stackrel{(n)}{1})$ of the $n$th power of $\Delta^{(n)}(\{d X \otimes d Y-d Y \otimes d X\})$, where product in the $\mathrm{n}$ th tensor power $\otimes^{(N)} \mathcal{T}(\mathcal{L})$ is defined exactly as in the case $n=2$ 
in (25). Thus

$$
\begin{aligned}
& w_{n}^{(\sigma)} d T \otimes d T \cdots \otimes \stackrel{(n)}{d} T \\
& \left.=\left(\left(\Delta^{(n)}\left(\left\{d \widehat{A}^{(\sigma)} \otimes d \hat{A}^{\dagger(\sigma)}-d \hat{A}^{\dagger(\sigma)} \otimes d \hat{A}^{(\sigma)}\right\}\right)\right)^{n}\right)_{(1,1, \ldots,} \quad{ }_{(n)}^{(n)}\right) \\
& =\left(\left(\sum _ { 1 \leq j < k \leq n } \left(1_{\mathcal{T}(\mathcal{L})} \otimes \cdots \otimes\left\{d \widehat{A}^{(\sigma)}\right\} \otimes \cdots \otimes\left\{d \widehat{A}^{\dagger(\sigma)}\right\} \otimes \cdots \otimes 1_{\mathcal{T}(\mathcal{L})}^{(n)}\right.\right.\right. \\
& \left.\left.\left.-1_{\mathcal{T}(\mathcal{L})} \otimes \cdots \otimes\left\{d \hat{A}^{(j)}\right\} \otimes \cdots \otimes\left\{d \widehat{A}^{(\sigma)}\right\} \otimes \cdots \otimes 1_{\mathcal{T}(\mathcal{L})}^{(n)}\right)\right)^{n}\right)_{\left(1,1, \ldots, 1^{(n)}\right)}
\end{aligned}
$$

This calculation of $w_{n}^{(\sigma)}$ can be finished using some combinatorics. We do that in the following two sections.

\section{Some background about Eulerian and Euler numbers}

In this section, we present several lemmas about Euler numbers, Eulerian numbers, Euler polynomials and forth-back permutations, as we call them. These combinatorial results are of sufficient general nature to be of interest elsewhere. A view of the provided lemmas appear as exercises in [16], but are still proven here for the sake of completeness. Additional explanations to the used methods and many similar results can be found in $[16,17]$.

A permutation $\mathfrak{s}$ in the symmetric group $\mathcal{S}_{n}$ is a zigzag permutation (misleadingly also called alternating permutation) if $\mathfrak{s}(1)>\mathfrak{s}(2)<\mathfrak{s}(3)>\mathfrak{s}(4)<\cdots$. In other words, $\mathfrak{s}$ is zigzag if $\mathfrak{s}(1)>\mathfrak{s}(2)$ and

$$
\text { either } \quad \mathfrak{s}(j-1)<\mathfrak{s}(j)>\mathfrak{s}(j+1) \quad \text { or } \quad \mathfrak{s}(j-1)>\mathfrak{s}(j)<\mathfrak{s}(j+1)
$$

for all $j \in\{2,3 \ldots, n-1\}$. If we have the initial condition $\mathfrak{s}(1)<\mathfrak{s}(2)$, instead of $\mathfrak{s}(1)>\mathfrak{s}(2)$, we may call $\mathfrak{s}$ zagzig. The number of all zigzag permutations in $\mathcal{S}_{n}$ is the Euler zigzag number $A_{n}$. These numbers occur in many places, for instance, as the coefficients of $\frac{z^{2 n}}{(2 n) !}$ in the Maclaurin series of $\sec (z)+\tan (z)$. In this paper, we meet them as the number of forth-back permutations, as we call them. These are the permutations $\mathfrak{s} \in \mathcal{S}_{n}$ with

$$
\text { either } \mathfrak{s}^{-1}(j)<j>\mathfrak{s}(j) \text { or } \mathfrak{s}^{-1}(j)>j<\mathfrak{s}(j)
$$

for all $j \in\{1,2, \ldots, n\}$. As forth-back permutations do not contain cycles of odd length, $n$ must be even for there to exist forth-back permutations. If $n$ is even, say $n=2 m>0$, we have the following lemma: 
Lemma 8. The number of forth-back permutations in $\mathcal{S}_{2 m}$ is the Euler zigzag number $A_{2 m}$.

Proof. A bijection between the forth-back permutations $\mathfrak{s}$ and the zigzag permutations in $\mathcal{S}_{2 m}$ is obtained by applying the so-called transformation fundamentale [4]. To perform this transformation, we write $\mathfrak{s}$ in cycle notation

$$
\begin{aligned}
\mathfrak{s}= & \left(\mathfrak{s}_{1}, \mathfrak{s}_{2}, \ldots, \mathfrak{s}_{\ell_{2}-1}\right)\left(\mathfrak{s}_{\ell_{2}}, \mathfrak{s}_{\ell_{2}+1}, \ldots, \mathfrak{s}_{\ell_{3}-1}\right)\left(\mathfrak{s}_{\ell_{3}}, \mathfrak{s}_{\ell_{3}+1}, \ldots, \mathfrak{s}_{\ell_{4}-1}\right) \ldots \\
& \left(\mathfrak{s}_{\ell_{m}}, \mathfrak{s}_{\ell_{m}+1}, \ldots, \mathfrak{s}_{2 m}\right)
\end{aligned}
$$

This representation and the numbers $\mathfrak{s}_{j}$ are uniquely determined if we require that the first entry of every cycle is bigger than all other entries in that cycle, and also that $\mathfrak{s}_{1}<\mathfrak{s}_{\ell_{2}}<\mathfrak{s}_{\ell_{3}}<\cdots<\mathfrak{s}_{\ell_{m}}$. The new permutation $\overline{\mathfrak{s}}$ is then obtained by forgetting brackets and setting $\overline{\mathfrak{s}}(j):=\mathfrak{s}_{j}$. We just have to see that this actually yields a bijection $\mathfrak{s} \mapsto \overline{\mathfrak{s}}$ between forth-back and zigzag permutations. To do this we proceed as follows.

Assume first that $\mathfrak{s}$ is forth-back. Then all cycles necessarily have even length and the permutation $\overline{\mathfrak{s}}$ is obviously zigzag, $\mathfrak{s}_{1}>\mathfrak{s}_{2}<\mathfrak{s}_{3}>\mathfrak{s}_{4}<\cdots>\mathfrak{s}_{2 m}$. Conversely, let us show that every zigzag permutation $\overline{\mathfrak{s}}$ has a unique pre-image $\mathfrak{s}$, and that that pre-image is forth-back. To construct a pre-image $\mathfrak{s}$ of $\overline{\mathfrak{s}}$, we only need to find suitable numbers $\ell_{j}$, which indicate where we have to insert brackets into the sequence $\left(\mathfrak{s}_{1}, \mathfrak{s}_{2}, \ldots, \mathfrak{s}_{2}\right):=(\mathfrak{s}(1), \mathfrak{s}(2), \ldots, \mathfrak{s}(2 m))$ to actually get a preimage. However, if we have already found $\ell_{2}, \ell_{3}, \ldots, \ell_{j}$, then $\ell_{j+1}$ is necessarily the first index $x$ with $\mathfrak{s}_{x}>\mathfrak{s}_{\ell_{j}}$. Using this, we can construct a pre-image $\mathfrak{s}$ of $\overline{\mathfrak{s}}$ in $\mathcal{S}_{2 m}$, and it is uniquely determined. Moreover, if $\overline{\mathfrak{s}}$ is zigzag then this construction ensures that $\mathfrak{s}_{1}$ and the $\mathfrak{s}_{\ell_{j}}$ are peaks and their neighbors and $\mathfrak{s}_{2 m}$ are valleys. Since also $\mathfrak{s}_{1}>\mathfrak{s}_{\ell_{2}-1}, \mathfrak{s}_{\ell_{2}}>\mathfrak{s}_{\ell_{3}-1}, \ldots, \mathfrak{s}_{\ell_{m}}>\mathfrak{s}_{2 m}$, insertion of brackets before the peaks $\ell_{j}$ yields forth-back cycles in $\mathfrak{s}$.

With the bijection established, it is now clear that there are as many forth-back permutations as there are zigzag permutations in $\mathcal{S}_{2 m}$. This number is the Euler zigzag number $A_{2 m}$.

The number of forth-back permutations with just one cycle is given by the following lemma. If $\mathcal{C}_{n}$ denotes the subset of cyclic permutations in $\mathcal{S}_{n}$, we have:

Lemma 9. The number of forth-back permutations in $\mathcal{C}_{2 m}$ is $A_{2 m-1}$.

Proof. The cycle notation $\mathfrak{s}=\left(\mathfrak{s}_{1}, \mathfrak{s}_{2}, \ldots, \mathfrak{s}_{2 m}\right)$ of cyclic permutations $\mathfrak{s} \in \mathcal{S}_{2 m}$ is not uniquely determined, as one may rotate the entries cyclically. It becomes uniquely determined if we require that $\mathfrak{s}_{2 m}=2 \mathrm{~m}$. In this case, removal of the 
last entry yields a sequence $\left(\mathfrak{s}_{1}, \mathfrak{s}_{2}, \ldots, \mathfrak{s}_{2 m-1}\right)$ that is zagzig $\left(\right.$ with $\mathfrak{s}_{1}<\mathfrak{s}_{2}$ as $\mathfrak{s}_{2 m}$ was the biggest entry of $\left.\mathfrak{s}\right)$. If we define $\overline{\mathfrak{s}} \in \mathcal{S}_{2 m-1}$ by setting $\overline{\mathfrak{s}}(j):=\mathfrak{s}_{j}$, for $j=1,2, \ldots, 2 m-1$, we obtain a bijection $\mathfrak{s} \mapsto \overline{\mathfrak{s}}$ from the cyclic forth-back permutations in $\mathcal{S}_{2 m}$ to the zagzig permutations in $\mathcal{S}_{2 m-1}$. Indeed, every zagzig permutation $\overline{\mathfrak{s}}$ in $\mathcal{S}_{2 m-1}$ has the cycle $\mathfrak{s}:=(\overline{\mathfrak{s}}(1), \overline{\mathfrak{s}}(2), \ldots, \overline{\mathfrak{s}}(2 m-1), 2 m)$ as unique pre-image. The existence of this bijection shows that the number of cyclic forthback permutations in $\mathcal{S}_{2 m}$ is equal to the number of zagzig permutations in $\mathcal{S}_{2 m-1}$, which is $A_{2 m-1}$, as for zigzag permutations.

This enumerative result about cyclic forth-back permutations can also be applied to forth-back permutation with $k$ cycles of lengths $2 m_{1}, 2 m_{2}, \ldots, 2 m_{k}$ (necessarily all even). To formulate this, we denote with $\mathcal{C}_{n_{1}, n_{2}, \ldots, n_{k}}$ the set of all permutations in $\mathcal{S}_{n}$ with $k$ cycles of lengths $n_{1}, n_{2}, \ldots, n_{k}$, i.e. the set of permutations of typ $\left(n_{1}, n_{2}, \ldots, n_{k}\right)$, as we say. We also denote with $\left[\begin{array}{c}n \\ n_{1}, n_{2}, \ldots, n_{k}\end{array}\right]$ the number of unordered partitions $\left\{N_{1}, N_{2}, \ldots, N_{k}\right\}$ of the set $\{1,2, \ldots, n\}$ with $k$ blocks $N_{j}$ of sizes $\left|N_{j}\right|=n_{j}>0$. With this we get the following more general formula.

Lemma 10. If positive integers $m_{1} \leq m_{2} \leq \cdots \leq m_{k}$ with $m_{1}+m_{2}+\ldots+m_{k}=m$ are given, then the number of forth-back permutations in $\mathcal{S}_{2 m}$ with $k$ cycles of lengths $2 m_{1}, 2 m_{2}, \ldots, 2 m_{k}$ is

$$
\mid\left\{\mathfrak{s} \in \mathcal{C}_{2 m_{1}, 2 m_{2}, \ldots, 2 m_{k}} \mid \mathfrak{s} \text { is forth-back }\right\} \mid=\left[\begin{array}{c}
2 m \\
2 m_{1}, 2 m_{2}, \ldots, 2 m_{k}
\end{array}\right] \prod_{j=1}^{k} A_{2 m_{j}-1} .
$$

In particular,

$$
A_{2 m}=\sum\left[\begin{array}{c}
2 m \\
2 m_{1}, 2 m_{2}, \ldots, 2 m_{k}
\end{array}\right] \prod_{j=1}^{k} A_{2 m_{j}-1}
$$

where the sum runs over all partitions $m_{1}+m_{2}+\cdots+m_{k}$ of $m$, that is over all non-decreasing sequences $m_{1} \leq m_{2} \leq \cdots \leq m_{k}$ of positive integers of every possible length $k$ with $m_{1}+m_{2}+\cdots+m_{k}=m>0$.

Proof. If a partition $m_{1}+m_{2}+\cdots+m_{k}=m$ of the number $m$ is given, then there are $\left[\begin{array}{c}2 m \\ 2 m_{1}, 2 m_{2}, \ldots, 2 m_{k}\end{array}\right]$ partitions of the set $\{1,2, \ldots, 2 m\}$ into a set of $k$ blocks $N_{j}$ with sizes $2 m_{j}, j=1,2, \ldots, k$. The block $N_{j}$ can be turned into a cyclic forth-back permutation in $A_{2 m_{j}-1}$ many ways, by Lemma 9. Hence, we get the stated expression for the number of forth-back permutations of that type. 
Moreover, it is easy to see that the sum over all possible expressions of this form gives the number of all forth-back permutations, which is $A_{2 m}$ by Lemma 8 . Indeed, every forth-back permutations $\mathfrak{s}$ in $\mathcal{S}_{2 m}$, has a certain number $k$ of cycles and a certain type, certain lengths $2 m_{1}, 2 m_{2}, \ldots, 2 m_{k}$ of its cycles. In this respect, every partition $m_{1}+m_{2}+\cdots+m_{k}=m$ is possible. Hence, the sum covers all $A_{2 m}$ forth-back permutations, as stated.

In our investigations, we will also need to look at a certain notion of sign, denoted by $\operatorname{sn}(\mathfrak{s})$, for permutations $\mathfrak{s} \in \mathcal{S}_{n}$, defined by

$$
\operatorname{sn}(\mathfrak{s}):=\prod_{j=1}^{n}(-1)^{\operatorname{des}(j, \mathfrak{s}(j))}
$$

where

$$
\operatorname{des}(h, k):= \begin{cases}0 & \text { if } h \leq k \\ 1 & \text { if } h>k\end{cases}
$$

We want to show that $\sum_{\mathfrak{s} \in \mathcal{S}_{2 m}^{\neq}} \operatorname{sn}(\mathfrak{s})=(-1)^{m} A_{2 m}$, where $\mathcal{S}_{n}^{\neq}$denotes the set of fixed-point-free permutations in $\mathcal{S}_{n}$. To establish this and similar results, we need to introduce certain equivalence classes of permutations which are based on the notion of a transit of a permutation. We call $h \in\{1,2, \ldots, n\}$ a transit of the permutation $\mathfrak{s} \in \mathcal{S}_{n}$ if

$$
\text { either } \mathfrak{s}^{-1}(h)<h<\mathfrak{s}(h) \text { or } \quad \mathfrak{s}^{-1}(h)>h>\mathfrak{s}(h) .
$$

Let $\mathcal{S}_{n}^{T}$ denote the set of permutations in $\mathcal{S}_{n}$ which contain a transit, and let $\mathcal{C}_{n_{1}, n_{2}, \ldots, n_{k}}^{T}$ be the set of permutations in $\mathcal{C}_{n_{1}, n_{2}, \ldots, n_{k}}$ which contain a transit. Every permutation $\mathfrak{s}$ with transit contains a unique smallest transit $h$, say inside a cycle $\left(j_{1}, j_{2}, \ldots, \mathfrak{s}^{-1}(h), h, \mathfrak{s}(h), \ldots, j_{\ell}\right)$ of length $\ell$, which we may also write as

$$
j_{1} \longmapsto j_{2} \longmapsto \cdots \longmapsto \mathfrak{s}^{-1}(h) \longmapsto h \longmapsto \mathfrak{s}(h) \longmapsto \cdots \longmapsto j_{\ell} \longmapsto j_{1} .
$$

We obtain a permutation $\mathfrak{s}^{\prime}$ of $\{1,2, \ldots, n\} \backslash\{h\}$ by replacing the chain of assignments $\mathfrak{s}^{-1}(h) \mapsto h \mapsto \mathfrak{s}(h)$ with the shorter chain $\mathfrak{s}^{-1}(h) \longmapsto \mathfrak{s}(h)$. Hence, the new permutation $\mathfrak{s}^{\prime}$ contains the cycle

$$
j_{1} \longmapsto j_{2} \longmapsto \cdots \longmapsto \mathfrak{s}^{-1}(h) \longmapsto \mathfrak{s}(h) \longmapsto \cdots \longmapsto j_{\ell} \longmapsto j_{1} .
$$

We define an equivalence relation $\sim$ on the $\operatorname{set} \mathcal{S}_{n}^{T}$. For two permutations $\mathfrak{s}$ and $\mathfrak{r}$ with transit, we write $\mathfrak{s} \sim \mathfrak{r}$ if and only if $\mathfrak{s}$ and $\mathfrak{r}$ have the same smallest transit $h$, if $\mathfrak{s}^{\prime}=\mathfrak{r}^{\prime}$ and if the smallest transit $h$ is missing from the same cycle in $\mathfrak{s}^{\prime}$ as in $\mathfrak{r}^{\prime}$. The equivalence class of $\mathfrak{s}$ is denoted as $[\mathfrak{s}]$. The equivalence relation $\sim$ can also be restricted to the sets of the form $\mathcal{C}_{n_{1}, n_{2}, \ldots, n_{k}}^{T}$. We have $[\mathfrak{s}] \subseteq \mathcal{C}_{n_{1}, n_{2}, \ldots, n_{k}}^{T}$ whenever $\mathfrak{s} \in \mathcal{C}_{n_{1}, n_{2}, \ldots, n_{k}}^{T}$. 
Example 11. In the 8 -cycle $\mathfrak{s}:=(4,1,8,2,6,7,5,3)$ the number 5 is the smallest transit, a downwards transit in this case, as $7>5>3$. If we remove it from the cycle, and reinsert the 5 as a transit in all possible ways into the remaining 7 -cycle $(4,1,8,2,6,7,3)$, we get four permutations. The 5 would not be a transit between 4 and 1 , but can be inserted between 1 and 8 , yielding $(4,1,5,8,2,6,7,3)$. Similarly, we also obtain $(4,1,8,5,2,6,7,3),(4,1,8,2,5,6,7,3)$ and the original permutation $(4,1,8,2,6,7, \mathbf{5}, 3)$. These four 8 -cycles form the equivalence class [s] of $\mathfrak{s}$ with respect to $\sim$. Interestingly, two of the four 8-cycles contain the number 5 as upwards transit, and their sign is opposite to the sign of the other two 8-cycles with 5 as downwards transit, as one can easily check. The situation is illustrated in Figure 1.

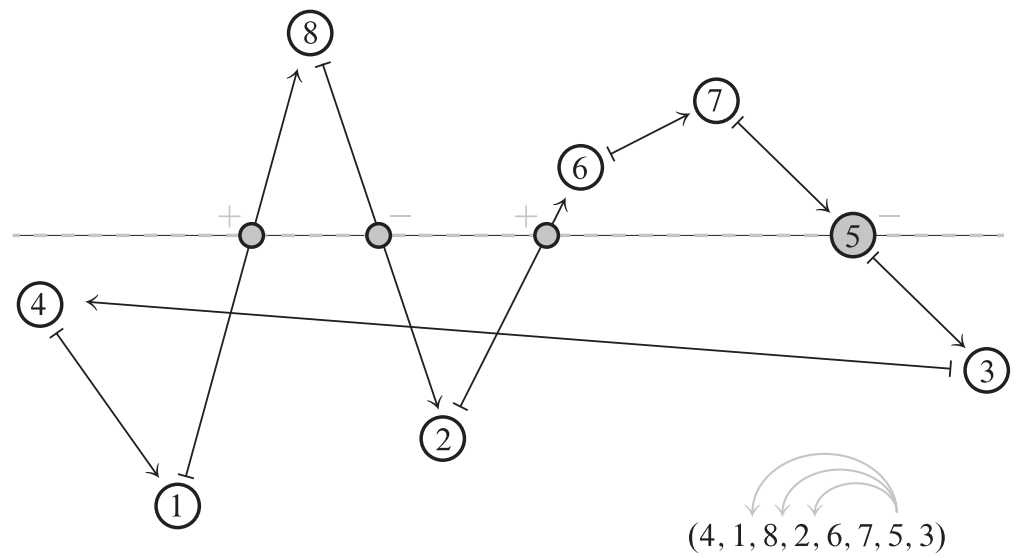

Figure 1 . The cycle $(4,1,8,2,6,7,5,3)$ with smallest transit $h=5$.

The observation about the sign of the elements in equivalence classes that we made in this example is no coincidence. We have the following lemma.

Lemma 12. If a permutation $\mathfrak{s}$ contains a transit, then there is an even number of elements in its equivalence class [s]. One half of them have negative sign, and one half have positive sign.

Proof. Assume the cycle that contains the smallest transit $h$ of $\mathfrak{s}$ is denoted as in equation (64). If we walk once around the shortened cycle of $\mathfrak{s}^{\prime}$ in equation (65) and observe the indices $j_{1}, j_{2}, j_{3}, \ldots$ as a kind of altitude, then we will cross the altitude $h$ as many times upwards (from below $h$ to above $h$ ) as downwards (from above $h$ to below $h$ ), see Figure 1. Hence, there are as many ways to reinsert 
$h$ as an upwards transit and as a downwards transit. Therefore, one half of the permutations that we obtain have positive sign, and one half have negative sign. The equivalence class [ $\mathfrak{s}]$ of $\mathfrak{s}$ is as claimed.

With the help of Lemma 12, we can now prove the following theorem.

Theorem 13. Let a partition $n_{1}+n_{2}+\ldots+n_{k}=n$ with $2 \leq n_{1} \leq n_{2} \leq \cdots \leq n_{k}$ be given. Then,

$$
\sum_{\mathfrak{s} \in \mathbb{C}_{n_{1}, n_{2}, \ldots, n_{k}} \operatorname{sn}(\mathfrak{s})}=\left\{\begin{array}{cl}
(-1)^{\frac{n}{2}}\left[\begin{array}{c}
n \\
n_{1}, n_{2}, \ldots, n_{k}
\end{array}\right] \prod_{j=1}^{k} A_{n_{j}-1} & \text { if all } n_{j} \text { are even, } \\
0 & \text { otherwise. }
\end{array}\right.
$$

In particular,

$$
\sum_{\mathfrak{s} \in \mathcal{S}_{n}^{\neq}} \operatorname{sn}(\mathfrak{s})=\left\{\begin{array}{cl}
(-1)^{\frac{n}{2}} A_{n} & \text { if } n \text { is even, } \\
0 & \text { otherwise. }
\end{array}\right.
$$

Proof. We observe that we can cancel out all permutations in $\mathcal{C}_{n_{1}, n_{2}, \ldots, n_{k}}$ that contain a transit, that is, all elements of $\mathcal{C}_{n_{1}, n_{2}, \ldots, n_{k}}^{T}$. In fact, $\mathcal{C}_{n_{1}, n_{2}, \ldots, n_{k}}^{T}$ is partitioned into equivalence classes, and each of them cancels out by Lemma 12. The remaining elements of $\mathcal{C}_{n_{1}, n_{2}, \ldots, n_{k}}$ do not contain a transit. Hence, if there are any remaining permutations in $\mathcal{C}_{n_{1}, n_{2}, \ldots, n_{k}} \backslash \mathcal{C}_{n_{1}, n_{2}, \ldots, n_{k}}^{T}$, they must be forth-back permutations. In particular, in this case, all $n_{j}$ must necessarily be even. Now, Lemma 10 yields the first stated result, since all forth-back permutations in $\mathcal{S}_{n}^{\neq}$ have sign $(-1)^{\frac{n}{2}}$. The second formula follows from this and the second formula in Lemma 10, but it can also be deduced from Lemma 8 directly, after canceling out equivalence classes in $\mathcal{S}_{n}^{\neq}$.

For the number of partitions in some of the previous results, we also have the following well known formula, whose proof we present for completeness:

Lemma 14. We have

$$
\left[\begin{array}{c}
n \\
n_{1}, n_{2}, \ldots, n_{k}
\end{array}\right]=\left(\begin{array}{c}
n \\
n_{1}, n_{2}, \ldots, n_{k}
\end{array}\right) \frac{1}{k_{1} ! k_{2} ! \cdots k_{r} !}=\frac{n !}{n_{1} ! n_{2} ! \cdots n_{k} ! k_{1} ! k_{2} ! \ldots k_{r} !},
$$

where $k_{1}, k_{2}, \ldots, k_{r}$ are the multiplicities of the different elements in the multi-set $\left\{n_{1}, n_{2}, \ldots, n_{k}\right\}$. (For example, the elements in the multi-set $\{2,2,2,4,4\}$ have the multiplicities $k_{1}=3$ and $k_{2}=2$, and $r=2$.) 
Proof. Without loss of generality, we may assume that $n_{1} \leq n_{2} \leq \cdots \leq n_{k}$. There are $\left(\begin{array}{c}n \\ n_{1}, n_{2}, \ldots, n_{k}\end{array}\right):=\frac{n !}{n_{1} ! n_{2} ! \cdots n_{k} !}$ ordered partitions (sequences of blocks) with block sizes $n_{1}, n_{2}, \ldots, n_{k}$ (in that order). This number can also be generated by first choosing all $\left[\begin{array}{c}n \\ n_{1}, n_{2}, \ldots, n_{k}\end{array}\right]$ unordered partitions (sets of blocks) with block sizes $n_{1}, n_{2}, \ldots, n_{k}$, and then arranging each of them in all possible ways as a sequences of blocks, i.e. as ordered partition. Hence, for each unordered partition $\left\{N_{1}, N_{2}, \ldots, N_{k}\right\}$, we have to see how many ways there are to arrange its blocks in a sequence with nondecreasing cardinalities (equal to the sequence $n_{1} \leq$ $n_{2} \leq \cdots \leq n_{k}$ ). Ambiguities in this order of the blocks are only given for blocks of equal size, which correspond to multiplicities of the elements in the multi-set $\left\{n_{1}, n_{2}, \ldots, n_{k}\right\}$. Hence, the number of ways is always $k_{1} ! k_{2} ! \ldots k_{r} !$, where $k_{1}, k_{2}, \ldots, k_{r}$ are the multiplicities of the different elements in the multi-set $\left\{n_{1}, n_{2}, \ldots, n_{k}\right\}$. Combining this factor with the number of unordered partitions yields the relation

$$
\left[\begin{array}{c}
n \\
n_{1}, n_{2}, \ldots, n_{k}
\end{array}\right] k_{1} ! k_{2} ! \cdots k_{r} !=\left(\begin{array}{c}
n \\
n_{1}, n_{2}, \ldots, n_{k}
\end{array}\right),
$$

which proves the lemma.

In this paper we will also consider the number of descends of sequences $\left(j_{1}, j_{2}, \ldots, j_{n}\right)$ of $n \geq 1$ integers, that is, the number

$$
\operatorname{des}\left(j_{1}, j_{2}, \ldots, j_{n}\right):=\left|\left\{\ell \in\{1,2, \ldots, n-1\} \mid j_{\ell}>j_{\ell+1}\right\}\right|,
$$

which generalizes $\operatorname{des}(h, k) \in\{0,1\}$ in (62). The number of permutations $\mathfrak{s} \in \mathcal{S}_{n}$ for which

$$
\operatorname{des}(\mathfrak{s}(1), \mathfrak{s}(2), \ldots, \mathfrak{s}(n))=j
$$

is the so-called Eulerian number $\left(\begin{array}{l}n \\ j\end{array}\right)$. We follow [16] in taking this as the definition of the Eulerian numbers, but Eulerian numbers also count various other kinds of objects, see [16]. The corresponding generating function is the so-called Euler polynomial

$$
S_{n}(\tau):=\sum_{\mathfrak{s} \in S_{n}} \tau^{\operatorname{des}(\mathfrak{s}(1), \mathfrak{s}(2), \ldots, \mathfrak{s}(n))}=\sum_{j=0}^{n-1}\left\langle\begin{array}{l}
n \\
j
\end{array}\right) \tau^{j} .
$$

In this paper, we will also need the closely related number of cyclic descends, defined by

$$
\operatorname{cdes}\left(j_{1}, j_{2}, \ldots, j_{n}\right):=\operatorname{des}\left(j_{1}, j_{2}, \ldots, j_{n}, j_{1}\right),
$$

which has the following statistic [16, Exercise1.11]. 
Lemma 15. All permutations $\mathfrak{s} \in \mathcal{S}_{n}$ have $0<\operatorname{cdes}(\mathfrak{s}(1), \mathfrak{s}(2), \ldots, \mathfrak{s}(n))<n$. For $0<j<n$, the number of permutations $\mathfrak{s} \in \mathcal{S}_{n}$ with exactly $j$ cyclic descents is

$$
\left|\left\{\mathfrak{s} \in \mathcal{S}_{n} \mid \operatorname{cdes}(\mathfrak{s}(1), \mathfrak{s}(2), \ldots, \mathfrak{s}(n))=j\right\}\right|=n\left\langle\begin{array}{c}
n-1 \\
j-1
\end{array}\right\rangle .
$$

In particular,

$$
\sum_{\mathfrak{s} \in S_{n}} \tau^{\operatorname{cdes}(\mathfrak{s}(1), \mathfrak{s}(2), \ldots, \mathfrak{s}(n))}=n \tau S_{n-1}(\tau) .
$$

Proof. Assume $\mathfrak{s} \in \mathcal{S}_{n}$ with $\operatorname{cdes}(\mathfrak{s}(1), \mathfrak{s}(2), \ldots, \mathfrak{s}(n))=j$. Since every sequence of distinct integers has at least one cyclic descent and one cyclic ascent, $j$ cannot be 0 or $n$, and $0<j<n$ as claimed. Now, let $M$ be such that $\mathfrak{s}(M)$ is the biggest entry of the sequence $(\mathfrak{s}(1), \mathfrak{s}(2), \ldots, \mathfrak{s}(n))$. We construct a new shorter sequence

$$
\overline{\mathfrak{s}}:=(\mathfrak{s}(M+1), \mathfrak{s}(M+2), \ldots, \mathfrak{s}(n), \mathfrak{s}(1), \mathfrak{s}(2), \ldots, \mathfrak{s}(M-1))
$$

by removing $\mathfrak{s}(M)$ and gluing together the remaining halves in opposite order. Obviously, $\overline{\mathfrak{s}}$ has exactly $j-1$ descends. If we first rotate the entries of the sequence $(\mathfrak{s}(1), \mathfrak{s}(2), \ldots, \mathfrak{s}(n))$ and then remove the biggest entry, we still get the same sequence $\overline{\mathfrak{s}}$ in the same way. This idea shows that removal of the biggest entry yields an $n$ to 1 correspondence $\mathfrak{s} \mapsto \overline{\mathfrak{s}}$ between the permutations in $\mathcal{S}_{n}$ with $j$ cyclic descends and the permutations in $\mathcal{S}_{n-1}$ with $j-1$ descends. Hence, there are $n\left(\begin{array}{l}n-1 \\ j-1\end{array}\right)$ permutations in $\mathcal{S}_{n}$ with exactly $j$ cyclic descends. In particular, this number is the coefficient of $\tau^{j}$ in both polynomial on the left of (71) and the polynomial on the right. So these polynomials are equal.

One can also prove the following lemma, which might be useful in calculations similar to the ones in our paper [16, Excercise 1.7].

Lemma 16. The number of permutations $\mathfrak{s} \in \mathcal{S}_{n}$ with $\mathfrak{s}(x)<x$ for exactly $j$ points $x \in\{1,2, \ldots, n\}$ is the Eulerian number $\left\langle\begin{array}{l}n \\ j\end{array}\right)$ and the corresponding generating function is the Euler polynomial.

\section{Moments of quantum Lévy areas}

To evaluate the moments $\mathbb{E}\left[\widehat{\mathcal{B}}_{[a, b)}^{(\sigma)}\right]^{n}$, we need to calculate the number $w_{n}^{(\sigma)}$, as explained in (54). By (57), we have

$$
\left.w_{n}^{(\sigma)} d T \otimes d T \cdots \otimes \stackrel{(n)}{d} T=\left(\left(\sum_{h \neq k}(-1)^{\operatorname{des}(h, k)} R_{h, k}\right)^{n}\right)_{(1,1, \ldots, 1}^{(n)}, \underset{1}{(n)}\right)
$$


with

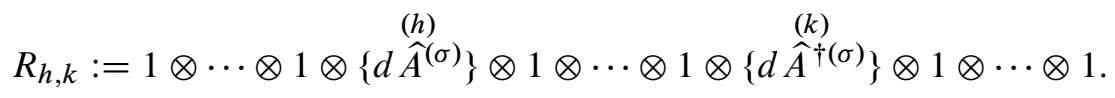

As in the previous section,

$$
\operatorname{des}(h, k):= \begin{cases}0 & \text { if } h \leq k, \\ 1 & \text { if } h>k,\end{cases}
$$

and the $n$th power is based on the sticky shuffle product in $\mathcal{T}(\mathcal{L})$ and its extension to the $\mathrm{n} t h$ tensor power $\otimes^{(N)} \mathcal{T}(\mathcal{L})$, as described in $(25)$ for $n=2$.

If we set $e:=(h, k)$, then we may also write $R_{e}$ for $R_{h, k}$ and $\operatorname{des}(e)$ for des $(h, k)$. Using distributivity, this yields

$$
w_{n}^{(\sigma)} d T \otimes d T \cdots \otimes \stackrel{(n)}{d T}=\left(\sum \prod_{\ell=1}^{n}(-1)^{\operatorname{des}\left(e_{\ell}\right)} R_{e_{\ell}}\right)_{(1,1, \ldots, 1}{ }_{(n)},
$$

where the sum runs over all $n$-tuples $\left(e_{1}, e_{2}, \ldots, e_{n}\right)$ of pairs $(h, k)$ with $h \neq k$. We may imagine each pair $e_{\ell}=\left(h_{\ell}, k_{\ell}\right)$ as a directed edge, an arc, from $h_{\ell}$ to $k_{\ell}$. Each $n$-tuples $\left(e_{1}, e_{2}, \ldots, e_{n}\right)$ is then a directed labeled multigraph, or digraph, on the vertex set $V:=\{1,2, \ldots, n\}$. It is important to keep track of the indices $\ell$ as labels of the arcs $e_{\ell}$, because our product is not commutative,

$$
d \hat{A}^{(\sigma)} d \hat{A}^{\dagger(\sigma)}=\sigma_{+} d T \quad \text { and } \quad d \hat{A}^{\dagger(\sigma)} d \hat{A}^{(\sigma)}=\sigma_{-} d T,
$$

and

$$
\left(\left\{d \hat{A}^{(\sigma)}\right\}\left\{d \hat{A}^{\dagger(\sigma)}\right\}\right)_{(1)}=\sigma_{+} d T \quad \text { and } \quad\left(\left\{d \hat{A}^{\dagger(\sigma)}\right\}\left\{d \hat{A}^{(\sigma)}\right\}\right)_{(1)}=\sigma_{-} d T .
$$

For example, in the case $n=4$, the two $\operatorname{arcs} e_{1}=(1,2)$ and $e_{2}=(2,3)$ contribute

$$
\begin{aligned}
& \left(R_{e_{1}} R_{e_{2}}\right)_{(1,1,1,1)} \\
& =\left(\left(\left\{d \hat{A}^{(\sigma)}\right\} \otimes\left\{d \hat{A}^{\dagger(\sigma)}\right\} \otimes 1 \otimes 1\right)\left(1 \otimes\left\{d \widehat{A}^{(\sigma)}\right\} \otimes\left\{d \hat{A}^{\dagger(\sigma)}\right\} \otimes 1\right)\right)_{(1,1,1,1)} \\
& =\left(\left\{d \widehat{A}^{(\sigma)}\right\} 1\right)_{(1)} \otimes\left(\left\{d \hat{A}^{\dagger(\sigma)}\right\}\left\{d \widehat{A}^{(\sigma)}\right\}\right)_{(1)} \otimes\left(1\left\{d \hat{A}^{\dagger(\sigma)}\right\}\right)_{(1)} \otimes(1 \cdot 1)_{(1)} \\
& =\sigma_{-} d \widehat{A}^{(\sigma)} \otimes d T \otimes d \hat{A}^{\dagger(\sigma)} \otimes 1,
\end{aligned}
$$

while if the labels 1 and 2 are exchanged, $e_{1}=(2,3)$ and $e_{2}=(1,2)$, we get

$$
\begin{aligned}
& \left(R_{e_{1}} R_{e_{2}}\right)_{(1,1,1,1)} \\
& =\left(\left(1 \otimes\left\{d \widehat{A}^{(\sigma)}\right\} \otimes\left\{d \hat{A}^{\dagger(\sigma)}\right\} \otimes 1\right)\left(\left\{d \widehat{A}^{(\sigma)}\right\} \otimes\left\{d \hat{A}^{\dagger(\sigma)}\right\} \otimes 1 \otimes 1\right)\right)_{(1,1,1,1)} \\
& =\sigma_{+} d \widehat{A}^{(\sigma)} \otimes d T \otimes d \hat{A}^{\dagger(\sigma)} \otimes 1 .
\end{aligned}
$$


In order to calculate the coefficient $w_{n}^{(\sigma)}$ of $d T \otimes d T \otimes \cdots \otimes d T$ in (73), we need to retain only those summands in (76) that contribute scalar multiple of $d T \otimes d T \otimes \cdots \otimes d T$. We may discard other summands. Hence, we do not have to sum over all digraphs $\left(e_{1}, e_{2}, \ldots, e_{n}\right)$. To see which ones we have to retain, let us assume that $\left(e_{1}, e_{2}, \ldots, e_{n}\right)$ yields a multiple of $d T \otimes d T \otimes \cdots \otimes d T$ in (76). Since the $n$ copies of $d \widehat{A}^{(\sigma)}$ and $n$ copies of $d \hat{A}^{\dagger(\sigma)}$ in the unexpanded product $\prod_{\ell=1}^{n} R_{e_{\ell}}$ must yield $n$ copies of $d T$, one in each possible position, each vertex of the digraph $\left(e_{1}, e_{2}, \ldots, e_{n}\right)$ must have exactly one incoming arc and one outgoing arc. Thus, $\left(e_{1}, e_{2}, \ldots, e_{n}\right)$ must consist of disjoint cyclically oriented cycles that cover $V$. This allows us to view each $\operatorname{arc} e_{\ell}=\left(h_{\ell}, k_{\ell}\right)$ as the assignment of a function value, $h_{\ell} \mapsto k_{\ell}=: \mathfrak{s}\left(h_{\ell}\right)$. We obtain a fixed-point-free permutation $\mathfrak{s}$ on $V=\{1,2, \ldots, n\}$. We obtain a second permutation $\mathfrak{l}$ on $V$ by assigning to each label $\ell \in V$ the vertex $h_{\ell}$ from which the $\operatorname{arc} e_{\ell}=\left(h_{\ell}, k_{\ell}\right)$ originates, $\ell \mapsto h_{\ell}=: \mathfrak{l}(\ell)$. The pair $(\mathfrak{l}, \mathfrak{s})$ of permutations, $\mathfrak{l}$ in $\mathcal{S}_{n}$ and $\mathfrak{s}$ in the set $\mathcal{S}_{n}^{\neq}$of fixed-point-free permutations of $V=\{1,2, \ldots, n\}$, contains the full information about $\left(e_{1}, e_{2}, \ldots, e_{n}\right)$. Our construction describes a bijection $\left(e_{1}, e_{2}, \ldots, e_{n}\right) \longmapsto(\mathfrak{l}, \mathfrak{s})$ from the set of digraphs $\left(e_{1}, e_{2}, \ldots, e_{n}\right)$ that contribute a multiple of $d T \otimes d T \otimes \cdots \otimes d T$ onto the set $\mathcal{S}_{n} \times \mathcal{S}_{n}^{\neq}$. The edges $e_{\ell}$ of the digraph $\left(e_{1}, e_{2}, \ldots, e_{n}\right)$ can be recovered from $\mathfrak{s}$ and $\mathfrak{l}$ through the formula

$$
e_{\ell}=(\mathfrak{l}(\ell), \mathfrak{s}(\mathfrak{l}(\ell))),
$$

which describes the inverse bijection $(\mathfrak{l}, \mathfrak{s}) \mapsto\left(e_{1}, e_{2}, \ldots, e_{n}\right)$. With this, the term $w_{n}^{(\sigma)} d T \otimes d T \otimes \cdots \otimes d T$ in (76) can be calculated as

$$
\begin{aligned}
& w_{n}^{(\sigma)} d T \otimes d T \otimes \cdots \otimes d T
\end{aligned}
$$

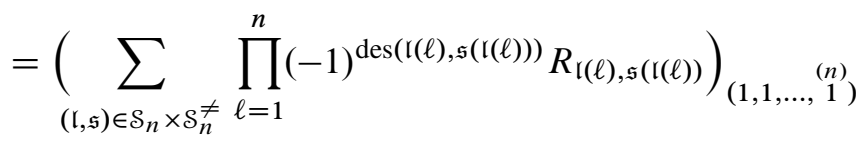

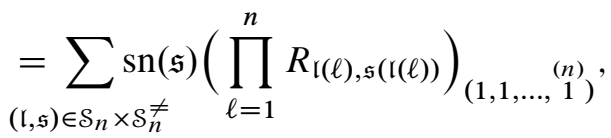

where, for every $\mathfrak{l} \in \mathcal{S}_{n}$,

$$
\operatorname{sn}(\mathfrak{s}):=\prod_{j=1}^{n}(-1)^{\operatorname{des}(j, \mathfrak{s}(j))}=\prod_{\ell=1}^{n}(-1)^{\operatorname{des}(r(\ell), \mathfrak{s}(r(\ell))} .
$$

We have to consider the product $\prod_{\ell=1}^{n} R_{\mathfrak{l}(\ell), \mathfrak{s}(\mathfrak{l}(\ell))}$, for every fixed $(\mathfrak{l}, \mathfrak{s}) \in$ $\mathcal{S}_{n} \times \mathcal{S}_{n}^{\neq}$. In this product, one $d T$ is produced in each position $j$, and it comes either with the scalar factor $\sigma_{+}$or with $\sigma_{-}$. If $d T$ arrives as $d \hat{A}^{(\sigma)} d \hat{A}^{\dagger(\sigma)}$ then we get $\sigma_{+}$ 
as scalar factor, if it arrives as $d \hat{A}^{\dagger(\sigma)} d \widehat{A}^{(\sigma)}$ then we get $\sigma_{-}$. To examine how the $d T$ in position $j$ arrives, let $\ell_{1}:=\mathfrak{l}^{-1}(j)$ and $\ell_{2}=\mathfrak{l}^{-1}\left(\mathfrak{s}^{-1}(j)\right)$, then $R_{\mathfrak{l}\left(\ell_{1}\right), \mathfrak{s}\left(r\left(\ell_{1}\right)\right)}$ contributes a $d \widehat{A}^{(\sigma)}$ in position $j$ as $\ell_{1}$ th factor, and $R_{\mathfrak{l}\left(\ell_{2}\right), \mathfrak{s}\left(\left[\left(\ell_{2}\right)\right)\right.}$ contributes a $d \hat{A}^{\dagger(\sigma)}$ in position $j$ as $\ell_{2}$ th factor. So, if $\left.\mathfrak{l}^{-1}\left(\mathfrak{s}^{-1}(j)\right)\right)>\mathfrak{l}^{-1}(j)$ then $\ell_{2}>\ell_{1}$ and the $d \hat{A}^{\dagger(\sigma)}$ comes after the $d \hat{A}^{(\sigma)}$, yielding a $\sigma_{+}$as scalar factor. In general, the scalar factor of the $d T$ in position $j$ is $\sigma_{-}\left(\sigma_{+} / \sigma_{-}\right)^{\operatorname{des}\left(I^{-1}\left(\mathfrak{s}^{-1}(j)\right), \mathfrak{I}^{-1}(j)\right)}$. Therefore,

$$
w_{n}^{(\sigma)}=\underset{(\mathfrak{l}, \mathfrak{s}) \in \mathcal{S}_{n} \times \mathcal{S}_{n}^{\neq}}{\sigma_{n}^{n}} \operatorname{sn}(\mathfrak{s}) \prod_{j=1}^{n}\left(\sigma_{+} / \sigma_{-}\right)^{\operatorname{des}\left(\mathfrak{l}^{-1}\left(\mathfrak{s}^{-1}(j)\right), \mathfrak{r}^{-1}(j)\right)} .
$$

We substitute $\mathfrak{s}(j)$ for $j$ and $\mathfrak{l}^{-1}$ for $\mathfrak{l}$, and obtain the following theorem.

Theorem 17. We have

$$
w_{n}^{(\sigma)}=\sigma_{-}^{n} \sum_{\mathfrak{s} \in \mathcal{S}_{n}^{\neq}} \operatorname{sn}(\mathfrak{s}) \sum_{\mathfrak{l} \in \mathcal{S}_{n}} \prod_{j=1}^{n} \tau^{\operatorname{des}(\mathfrak{l}(j), \mathfrak{l}(\mathfrak{s}(j)))},
$$

where $\tau:=\sigma_{+} / \sigma_{-}$.

In this expression for $w_{n}^{(\sigma)}$ there are many terms that cancel against each other when we carry out the sum. To remove these unnecessary summands and bundle together equal terms, we first study the inner sum

$$
w_{n}^{\mathfrak{s}}(\tau):=\sum_{\mathfrak{r} \in S_{n}} \prod_{j=1}^{n} \tau^{\operatorname{des}(\mathfrak{l}(j), \mathfrak{l}(\mathfrak{s}(j)))},
$$

for a fixed $\mathfrak{s} \in \mathcal{S}_{n}^{\neq}$. Initially, for simplicity, also assume that there is only one cycle, of length $n$ in $\mathfrak{s}$. In cycle notation, $\mathfrak{s}=\left(\mathfrak{s}_{1}, \mathfrak{s}_{2}, \ldots, \mathfrak{s}_{n}\right)$ with $\mathfrak{s}_{2}=\mathfrak{s}\left(\mathfrak{s}_{1}\right)$, $\mathfrak{s}_{3}=\mathfrak{s}\left(\mathfrak{s}_{2}\right)$, etc. In this particular case, by Lemma 15 ,

$$
\begin{aligned}
w_{n}^{\mathfrak{s}}(\tau) & =\sum_{\mathfrak{r} \in S_{n}} \prod_{\ell=1}^{n} \tau^{\operatorname{des}\left(\mathfrak{l}\left(\mathfrak{s}_{\ell}\right), \mathfrak{l}\left(\mathfrak{s}_{\ell+1}\right)\right)} \\
& =\sum_{\mathfrak{l} \in S_{n}} \tau^{\operatorname{cdes}\left(\mathfrak{l}\left(\mathfrak{s}_{1}\right), \mathfrak{l}\left(\mathfrak{s}_{2}\right), \ldots, \mathfrak{l}\left(\mathfrak{s}_{n}\right)\right)} \\
& =\sum_{\mathfrak{r} \in S_{n}} \tau^{\operatorname{cdes}(\mathfrak{r}(1), \mathfrak{r}(2), \ldots, \mathfrak{r}(n))} \\
& =n \tau S_{n-1}(\tau)
\end{aligned}
$$

where $\mathcal{S}_{n}(\tau)$ is the Euler polynomial and $\operatorname{cdes}(\mathfrak{r}(1), \mathfrak{r}(2), \ldots, \mathfrak{r}(n))$ denotes the number of descends of the sequence $(\mathfrak{r}(1), \mathfrak{r}(2), \ldots, \mathfrak{r}(n), \mathfrak{r}(1))$. 
Formula (86) holds only for cyclic permutations $\mathfrak{s} \in \mathcal{S}_{n}^{\neq}$. For the general case, suppose that $\mathfrak{s}$ has $k=k(\mathfrak{s})$ cycles of lengths $n_{1}, n_{2}, \ldots, n_{k}$, say where $2 \leq n_{1} \leq n_{2} \leq \cdots \leq n_{k}$ and $n_{1}+n_{2}+\cdots+n_{k}=n$. We say that $\left(n_{1}, n_{2}, \ldots, n_{k}\right)$ is the typ of $\mathfrak{s}$ and write $\mathfrak{s} \in \mathcal{C}_{n_{1}, n_{2}, \ldots, n_{k}}$. We have to split the product in the definition (85) of $w_{n}^{\mathfrak{s}}(\tau)$ into $k$ parts correspondingly. If $C_{\ell}$ denotes the set of the $n_{\ell}$ elements of the $\ell$ th cycle of the fixed given $\mathfrak{s} \in \mathcal{C}_{n_{1}, n_{2}, \ldots, n_{k}}$, then

$$
\prod_{j=1}^{n} \tau^{\operatorname{des}(\mathfrak{l}(j), \mathfrak{r}(\mathfrak{s}(j)))}=\prod_{\ell=1}^{k} \prod_{j \in C_{\ell}} \tau^{\operatorname{des}\left(\mathfrak{r}_{\ell}(j), \mathfrak{r}_{\ell}(\mathfrak{s}(j))\right)},
$$

where $\mathfrak{l}_{\ell}$ is the restriction of $\mathfrak{l}$ to $C_{\ell}$, so that $\mathfrak{l}=\mathfrak{l}_{1} \cup \mathfrak{l}_{2} \cup \cdots \cup \mathfrak{l}_{n}$. The range of each $\mathfrak{l}_{\ell}$ can be any subset $N_{\ell} \subseteq V$ of $n_{\ell}$ elements, provided only that all the subsets $N_{\ell}$ together form an ordered partition $\left(N_{1}, N_{2}, \ldots, N_{k}\right)$ of $V$. We want to describe the set $\delta_{n}$ of permutations $\mathfrak{l}$ in terms of smaller bijections $\mathfrak{l}_{\ell}: C_{\ell} \rightarrow N_{\ell}$. Let $\mathcal{N}$ denotes the set of all partitions $N:=\left(N_{1}, N_{2}, \ldots, N_{k}\right)$ of $V$ into $k$ blocks $N_{\ell}$ with $\left|N_{\ell}\right|=n_{\ell}$, let $B_{\ell}(N)$ be the set of bijections from $C_{\ell}$ to $N_{\ell}$, and let $B(N):=B_{1}(N) \times B_{2}(N) \times \cdots \times B_{n}(N)$. With this, the set of permutations $\mathcal{S}_{n}$ is partitioned as

$$
\mathcal{S}_{n}=\bigcup_{N \in \mathcal{N}}\left\{\mathfrak{l}_{1} \cup \mathfrak{l}_{2} \cup \cdots \cup \mathfrak{l}_{n} \mid\left(\mathfrak{l}_{1}, \mathfrak{l}_{2}, \ldots, \mathfrak{l}_{n}\right) \in B(N)\right\} .
$$

From that disjoint union we get

$$
\begin{aligned}
w_{n}^{\mathfrak{s}}(\tau) & =\sum_{N \in \mathcal{N}} \sum_{\mathfrak{l} \in B(N)} \prod_{\ell=1}^{k} \prod_{j \in C_{\ell}} \tau^{\operatorname{des}\left(\mathfrak{l}_{\ell}(j), \mathfrak{r}_{\ell}(\mathfrak{s}(j))\right)} \\
& =\sum_{N \in \mathcal{N}} \prod_{\ell=1}^{k} \sum_{\mathfrak{l}_{\ell} \in B_{\ell}(N)} \prod_{j \in C_{\ell}} \tau^{\operatorname{des}\left(\mathfrak{r}_{\ell}(j), \mathfrak{l}_{\ell}(\mathfrak{s}(j))\right)} .
\end{aligned}
$$

Here, for all $N \in \mathcal{N}$, the inner sum is

$$
\sum_{\mathfrak{l}_{\ell} \in B_{\ell}(N)} \prod_{j \in C_{\ell}} \tau^{\operatorname{des}\left(\mathfrak{r}_{\ell}(j), \mathfrak{r}_{\ell}(\mathfrak{s}(j))\right)}=n_{\ell} \tau S_{n_{\ell}-1}(\tau),
$$

by (86), because the names of the elements in $C_{\ell}$ and $N_{\ell}$ do not matter. Every fixed set $N_{\ell}$ of $n_{\ell}$ different numbers is linearly ordered and produces the same statistic for the cyclic descents, if we consider all sequences that can be arranged using all elements of $N_{\ell}$. Using

$$
|\mathcal{N}|=\left(\begin{array}{c}
n \\
n_{1}, n_{2}, \ldots, n_{k}
\end{array}\right):=\frac{n !}{n_{1} ! n_{2} ! \cdots n_{k} !},
$$


we obtain

$$
w_{n}^{\mathfrak{s}}(\tau):=\left(\begin{array}{c}
n \\
n_{1}, n_{2}, \ldots, n_{k}
\end{array}\right) \prod_{j=1}^{k} n_{j} \tau S_{n_{j}-1}(\tau),
$$

where $\left(n_{1}, n_{2}, \ldots, n_{k}\right)$ is still the typ of $\mathfrak{s}$, i.e. $\mathfrak{s} \in \mathcal{C}_{n_{1}, n_{2}, \ldots, n_{k}}$.

We can now calculate $w_{n}^{(\sigma)}$ out of (92) and Theorem 17. Since we have the disjoint union

$$
\begin{gathered}
\mathcal{S}_{n}^{\neq}=\bigcup \mathcal{C}_{n_{1}, n_{2}, \ldots, n_{k}}, \\
n_{1}+n_{2}+\cdots+n_{k}=n, \\
2 \leq n_{1} \leq n_{2} \leq \cdots \leq n_{k}
\end{gathered}
$$

we get

$$
\begin{aligned}
w_{n}^{(\sigma)} & =\sigma_{-}^{n} \sum_{\mathfrak{s} \in \mathcal{S}_{n}^{\neq}} \operatorname{sn}(\mathfrak{s})\left(\begin{array}{c}
n \\
n_{1}, n_{2}, \ldots, n_{k}
\end{array}\right) \prod_{\ell=1}^{k} n_{\ell} \tau S_{n_{\ell}-1}(\tau) \\
& =\sigma_{-}^{n} \sum_{\substack{n_{1}+n_{2}+\cdots+n_{k}=n, 2 \leq n_{1} \leq n_{2} \leq \cdots \leq n_{k}}} \sum_{n_{1}, n_{2}, \ldots, n_{k}} \operatorname{sn}(\mathfrak{s})\left(\begin{array}{c}
n \\
n_{1}, n_{2}, \ldots, n_{k}
\end{array}\right) \prod_{\ell=1}^{k} n_{\ell} \tau S_{n_{\ell}-1}(\tau) . \\
& =\sigma_{-}^{n} \sum_{\substack{n_{1}+n_{2}+\cdots+n_{k}=n, 2 \leq n_{1} \leq n_{2} \leq \cdots \leq n_{k}}}\left(\begin{array}{c}
n \\
n_{1}, n_{2}, \ldots, n_{k}
\end{array}\right)\left(\prod_{\ell=1}^{k} n_{\ell} \tau S_{n_{\ell}-1}(\tau)\right) \sum_{\mathfrak{s} \in \mathcal{C}_{n_{1}, n_{2}, \ldots, n_{k}}} \operatorname{sn}(\mathfrak{s}) .
\end{aligned}
$$

Now, Theorem 13 shows that $w_{n}^{(\sigma)}=0$ for odd $n$, and that for even $n, n=2 m$,

$$
\begin{gathered}
w_{2 m}^{(\sigma)}=(-1)^{m} \sigma_{-}^{2 m} \sum_{\substack{m_{1}+m_{2}+\cdots+m_{k}=m \\
1 \leq m_{1} \leq m_{2} \leq \cdots \leq m_{k}}}\left[\begin{array}{c}
2 m \\
2 m_{1}, 2 m_{2}, \ldots, 2 m_{k}
\end{array}\right]\left(\begin{array}{c}
2 m \\
2 m_{1}, 2 m_{2}, \ldots, 2 m_{k}
\end{array}\right) \\
\prod_{\ell=1}^{k} 2 m_{\ell} A_{2 m_{\ell}-1} \tau S_{2 m_{\ell}-1}(\tau) .
\end{gathered}
$$

Using Lemma 14, we obtain the following theorem:

Theorem 18. For odd $n, w_{n}^{(\sigma)}=0$. For even $n, n=2 m>0$, we have

$$
w_{2 m}^{(\sigma)}=(-1)^{m}(2 m) !^{2} \sum \frac{\sigma_{-}^{2 m-k} \sigma_{+}^{k}}{k_{1} ! k_{2} ! \cdots k_{r} !} \prod_{j=1}^{k} \frac{A_{2 m_{j}-1}}{2 m_{j}\left(2 m_{j}-1\right) !^{2}} S_{2 m_{j}-1}\left(\sigma_{+} / \sigma_{-}\right),
$$

where the sum runs over all partitions $m_{1}+m_{2}+\cdots+m_{k}=m$ with $1 \leq m_{1} \leq$ $m_{2} \leq \cdots \leq m_{k}$, and where $k_{1}, k_{2}, \ldots, k_{r}$ are the corresponding multiplicities of the different elements in the multi-set $\left\{m_{1}, m_{2}, \ldots, m_{k}\right\}$. 
From this and equation (54), we derive our final result:

Theorem 19. The nonzero moments of the quantum Lévy area $\widehat{\mathcal{B}}_{[a . b)}^{(\sigma)}$ are

$$
\begin{aligned}
\mathbb{E} & {\left[\left(\widehat{\mathcal{B}}_{[a . b)}^{(\sigma)}\right)^{2 m}\right] } \\
& =(2 m) !(b-a)^{2 m} \sum \frac{\sigma_{-}^{2 m-k} \sigma_{+}^{k}}{k_{1} ! k_{2} ! \cdots k_{r} !} \prod_{j=1}^{k} \frac{A_{2 m_{j}-1}}{2 m_{j}\left(2 m_{j}-1\right) !^{2}} S_{2 m_{j}-1}\left(\sigma_{+} / \sigma_{-}\right),
\end{aligned}
$$

where the sum runs over all partitions $m_{1}+m_{2}+\cdots+m_{k}=m$ with $1 \leq m_{1} \leq$ $m_{2} \leq \cdots \leq m_{k}$, and where $k_{1}, k_{2}, \ldots, k_{r}$ are the corresponding multiplicities of the different elements in the multi-set $\left\{m_{1}, m_{2}, \ldots, m_{k}\right\}$. The $A_{n}$ are Euler zigzag numbers and the $S_{n}$ are Euler polynomials.

\section{The classical limit}

We calculate the limit of $\mathbb{E}\left[\left(\hat{\mathcal{B}}_{[a . b)}^{(\sigma)}\right)^{2 m}\right]$ as $\sigma \rightarrow \infty$, or equivalently, $\sigma_{+} \rightarrow \frac{1}{2}$, $\sigma_{-} \rightarrow \frac{1}{2}$. Putting $\sigma_{+}=\sigma_{-}=\frac{1}{2}$ in (96), we get

$$
\begin{aligned}
\lim _{\sigma \rightarrow \infty} \mathbb{E}\left[\left(\widehat{\mathcal{B}}_{[a . b)}^{(\sigma)}\right)^{2 m}\right] \\
\quad=(2 m) !\left(\frac{b-a}{2}\right)^{2 m} \sum \frac{1}{k_{1} ! k_{2} ! \cdots k_{r} !} \prod_{j=1}^{k} \frac{A_{2 m_{j}-1}}{2 m_{j}\left(2 m_{j}-1\right) !^{2}} S_{2 m_{j}-1}(1),
\end{aligned}
$$

where the sum runs over all partitions $m_{1}+m_{2}+\cdots+m_{k}=m$, and where $k_{1}$, $k_{2}, \ldots, k_{r}$ are the corresponding multiplicities of the elements of the multi-set $\left\{m_{1}, m_{2}, \ldots, m_{k}\right\}$. But, by the definition of the Euler polynomial,

$$
S_{n}(1):=\sum_{\mathfrak{s} \in \mathcal{S}_{n}} 1^{\operatorname{des}(\mathfrak{s}(1), \mathfrak{s}(2), \ldots, \mathfrak{s}(n))}=\left|\mathcal{S}_{n}\right|=n ! .
$$

So, using Lemma 10 and Lemma 14, we see that

$$
\begin{aligned}
\lim _{\sigma \rightarrow \infty} \mathbb{E}\left[\left(\widehat{\mathcal{B}}_{[a . b)}^{(\sigma)}\right)^{2 m}\right] & =(2 m) !\left(\frac{b-a}{2}\right)^{2 m} \sum \frac{1}{k_{1} ! k_{2} ! \cdots k_{r} !} \prod_{j=1}^{k} \frac{A_{2 m_{j}-1}}{\left(2 m_{j}\right) !} \\
& =\left(\frac{b-a}{2}\right)^{2 m} A_{2 m} .
\end{aligned}
$$

This result is in agreement with the main theorem in [11]. 


\section{References}

[1] N. Bourbaki, Elements of mathematics. Algebra, Part I: Chapters 1-3. Translated from the French. Hermann, Paris, and Addison-Wesley, Reading, MA, 1974. MR 0354207 Zbl 0281.00006

[2] P. B. Cohen, T. M. W. Eyre, and R. L.bHudson, Higher order Itô product formula and generators of evolutions and flows. Internat. J. Theoret. Phys. 34 (1995), no. 8, 1481-1486. Proceedings of the International Quantum Structures Association, Quantum Structures '94 (Prague, 1994). MR 1353690 Zbl 0850.81035

[3] S.Chen and R. L. Hudson, Some properties of quantum Lévy area in Fock and nonFock quantum stochastic calculus. Probab. Math. Statist. 33 (2013), no. 2, 425-434. MR 3158567 Zbl 1283.81099

[4] D. Foata and M. Schützenberger, Théorie géométrique des polynómes eulériens. Lecture Notes in Mathematics, 138. Springer-Verlag, Berlin etc., 1970. MR 0272642 Zbl 0214.26202

[5] M. Hoffman, The algebra of multiple harmonic series. J. Algebra 194 (1997), no. 2, 477-495. MR 1467164 Zbl 0881.11067

[6] R. L.Hudson, Sticky shuffle Hopf algebras and their stochastic representations. In H. Zhao and A. Truman (eds.), New trends in stochastic analysis and related topics. A volume in honour of Professor K. D. Elworthy. World Scientific, Hackensack, N.J., 2012, 165-181. MR 2920199 Zbl 1266.81123

[7] R. L. Hudson, Quantum Lévy area as quantum martingale limit. In L. Accardi and F. Fagnola (eds.), Quantum probability and related topics. Proceedings of the $32^{\text {nd }}$ Conference held in Levico Terme, May 29-June 4, 2011. Quantum Probability and White Noise Analysis, 29. World Scientific, Hackensack, N.J., 2013, 169-188. MR 3288045 Zbl 1327.81253

[8] R. L. Hudson and J. M. Lindsay, A non-commutative martingale representation theorem for non-Fock quantum Brownian motion. J. Funct. Anal. 61 (1985), no. 2, 202-221. MR 0786622 Zbl 0577.60055

[9] R. L. Hudson, U.Schauz, and Y.Wu, The moments of Lévy's area using a sticky shuffle Hopf algebra. Commun. Stoch. Anal. 11 (2017), no. 3, 287-299. MR 3734126

[10] R. L. Hudson and Y. Pei, On a causal quantum stochastic double product integral related to Lévy area. To appear in Ann. Inst. Henri Poincaré Comb. Phys. Interact.

[11] D. Levin and M. Wildon, A combinatorial method of calculating the moments of Lévy area. Trans. Amer. Math. Soc. 360 (2008), no. 12, 6695-6709. MR 2434307 Zbl 1152.60064

[12] P. Lévy, Le mouvement Brownien plan. Amer. J. Math. 62 (1940). 487-550. MR 0002734 Zbl 0024.13906 
[13] P. Lévy, Wiener's random function and other Laplacian functions. In J. Neyman (ed.), Proceedings of the second Berkeley symposium on mathematical statistics and probability. Held at the Statistical Laboratory, Department of Mathematics, University of California, July 31-August 12, 1950. University of California Press, Berkeley and Los Angeles, 1951, 171-187. MR 0044774 Zbl 0044.13802

[14] K. Newman and D. E. Radford, The cofree irreducible Hopf algebra on an algebra. Amer. J. Math. 101 (1979), no. 5, 1025-1045. MR 0546301 Zbl 0422.16003

[15] K. R. Parthasarathy, An introduction to quantum stochastic calculus. Monographs in Mathematics, 85. Birkhäuser Verlag, Basel, 1992. MR 1164866 Zbl 0751.60046

[16] T. K. Petersen, Eulerian numbers. With a foreword by R. Stanley. Birkhäuser Advanced Texts: Basler Lehrbücher. Birkhäuser/Springer, New York, 2015. MR 3408615 Zbl 1337.05001

[17] R. P. Stanley, A survey of alternating permutations. In R. A. Brualdi, S. Hedayat, H. Kharaghani, G. B. Khosrovshahi, and S. Shahriari (eds.), Combinatorics and graphs. Selected papers from the $20^{\text {th }}$ International Anniversary Conference (IPM 20) held in Tehran, May 15-21, 2009. Contemporary Mathematics, 531. American Mathematical Society, Providence, R.I., 2010, 165-196. MR 2757798 Zbl 1231.05288

(C) European Mathematical Society

Communicated by Frédéric Patras

Received January 30, 2017; revised May 14, 2017; accepted June 10, 2017

Robin Hudson, Department of Mathematical Sciences, Loughborough University, LE11 3TU,UK

e-mail: R.Hudson@1boro.ac.uk

Uwe Schauz, Department of Mathematical Sciences, Xi' an Jiaotong-Liverpool University, Suzhou, 215123, China

e-mail: uwe.schauz@xjtlu.edu.cn

Yue Wu, Technische Universität Berlin, Institut für Mathematik, Secr. MA 5-3, Straße des 17. Juni 136, DE-10623 Berlin, Germany

e-mail: wu@math.tu-berlin.de

School of Engineering, University of Edinburgh, EH9 3JL, UK

e-mail: yue.wu@ed.ac.uk 